\title{
Jugando con los polarizadores: una propuesta de un laboratorio didáctico interdisciplinario sobre la luz polarizada
}

\author{
Fabrizio Logiurato ${ }^{1}$ \\ Università di Trento \\ Italia \\ Wendy Roxana Llive Carrillo ${ }^{1}$ \\ Rocio Cecibel Jimenez Paute ${ }^{l}$ \\ Enith Vanessa Yánez Ramírez ${ }^{1}$ \\ Universidad Regional Amazónica Ikiam \\ Ecuador
}

\section{Resumen}

La polarización es una propiedad fundamental de la luz, con muchas aplicaciones prácticas en diferentes disciplinas. Sin embargo, el estudio de esta importante característica física a menudo se descuida en los programas de educación básica y superior. Esto es lamentable, porque la luz polarizada no solo es importante para comprender la naturaleza de las ondas electromagnéticas y el funcionamiento de muchos dispositivos tecnológicos de uso actual, sino que también, permite a los estudiantes realizar experiencias divertidas, de bajo costo y muy instructivas, que incluyen física, química, ingeniería, biología y también arte. Por lo tanto, se puede pensar en aprovechar estos aspectos para asegurarse de que el estudio de la física sea más interesante y que el proceso de aprendizaje sea más participativo. Con esto en mente, se ha desarrollado un laboratorio experimental interdisciplinario sobre la polarización de la luz, adaptable para estudiantes de nivel básico, secundario y universitario. En este artículo, se pretende describir algunos ejemplos de las actividades de este laboratorio y algunas nuevas ilustraciones de las propiedades de la luz polarizada. La característica principal de esta propuesta es el énfasis que se le da al lado artístico de

\footnotetext{
${ }^{+}$Playing with Polarizers: a Proposal for an Interdisciplinary Didactic Laboratory on Polarized Light

* Recebido: 18 de maio de 2021. Aceito: 18 de outubro de 2021.

${ }^{1}$ E-mails: fabrizio.logiurato@unitn.it; wendy.llive@ikiam.edu.ec; rocio.jimenez@ikiam.edu.ec; vanessa.yanez@ikiam.edu.ec
} 
la visualización científica y su interdisciplinariedad. Además, se dan explicaciones elementales de algunos fenómenos, a menudo dificiles de encontrar en español, con una breve introducción histórica.

Palabras-clave: Física Educativa; Interdisciplinariedad; Arte; Polarización de la Luz.

\begin{abstract}
Polarization is a fundamental property of light, with many practical applications in different disciplines. However, the study of this important characteristic of physics is often overlooked in secondary or high school programs. This is a pity, because polarized light is not only important to understand the nature of electromagnetic waves and how many technological devices in current use work, but it also allows the realization of fun, inexpensive and very instructive experiments, involving Physics, Chemistry, Engineering, Biology and also Art. So we can think of taking advantage of all this to make the study of physics more interesting and the process of learning much participative. With this in mind, we developed an interdisciplinary experimental laboratory on light polarization adaptable to basic, high school and undergraduate students. In this paper, we intend to describe some examples of activities presented in this laboratory and some new illustrations of the properties of polarized light. The feature of our work is the emphasis on the artistic side of scientific visualization and its interdisciplinarity. Furthermore, elementary explanations of some phenomena are given, often difficult to find in Spanish, with a brief historical introduction.
\end{abstract}

Keywords: Physics Education; Interdisciplinarity; Art; Light Polarization.

\title{
I. Introducción
}

La investigación científica actual se caracteriza por una interacción cada vez mayor entre diferentes disciplinas: solo por nombrar algunos ejemplos, el funcionamiento de los sistemas biológicos está fuertemente basado en la química y la física (DAVIDOVITS, 2008), así como la física es fundamental para comprender la formación de las estructuras atómicas y moleculares y el desarrollo de las reacciones químicas (ATKINS; DE PAULA, 2008). La medicina y la investigación en biología sería impensable sin las herramientas derivadas de la física moderna (SPLINTER, 2010): piensen en la resonancia magnética nuclear (RMN), la 
tomografía por emisión de positrones o PET, la ecografía, la electrocardiografía y la electroencefalografía, el uso de rayos $\mathrm{X}$ y láser, la espectroscopia para análisis químicos, en los diversos tipos de microscopios ópticos, electrónicos, de efecto túnel o de fuerza atómica. A la vez, las ciencias de la tierra y ambientales involucran prácticamente todas las ramas de la física y la química, llegando a la biología. Como áreas de investigación emergentes que se caracterizan por su interdisciplinariedad, podemos mencionar la biomimética (PASSINO, 2004), la ciencia que, a través de la imitación de la naturaleza, busca nuevas soluciones tecnológicas, y la biología cuántica (MCFADDEN; AL-KHALILI, 2014), la aplicación de efectos típicos de la mecánica cuántica (además de la química), como el entrelazamiento y el efecto túnel a las descripciones de diversos fenómenos biológicos, como, por ejemplo, la fotosíntesis, las mutaciones del ADN y la visión. Esta tendencia a la multidisciplinariedad e interdisciplinariedad está impulsando el cambio de muchos planes de estudios universitarios, especialmente en las facultades de ingeniería (ODEH; MCKENNA; ABU-MULAWEH, 2017), reflejando la necesidad no solo de una gran especialización de los estudiantes, sino también de una preparación más amplia, que abarque en su horizonte otras disciplinas, con las que el científico, al tener conocimiento de los conceptos fundamentales de estas, puede interactuar y dialogar con sus colegas de otras áreas y afrontar la solución de problemas reales, que a menudo no se limitan a una sola disciplina (KLAASSEN, 2018).

Por lo tanto, es deseable que las escuelas intermedias y secundarias también incluyan una mayor interacción entre las diferentes áreas científicas. Además, desde el punto de vista didáctico, al combinar en el momento de la docencia varias disciplinas, no solo técnicas y científicas, es posible captar más fácilmente el interés y la atención de los estudiantes, quienes logran una mejor comprensión y retención de los conceptos analizados. De hecho, con un enfoque multi e interdisciplinario, los conceptos y problemas se ubican más fácilmente en un contexto real, y los estudiantes están más estimulados para adquirir habilidades de síntesis y pensamiento crítico (SICHERL-KAFOLA; DENAC, 2010).

Teniendo en cuenta lo expresado previamente, en este artículo se quiere presentar un ejemplo de un laboratorio de física interdisciplinario, que involucra arte, historia, ciencia y técnica, basado en el estudio de la polarización de la luz.

La luz polarizada tiene varias aplicaciones importantes. Se utiliza en dispositivos tecnológicos desde la química hasta la medicina, desde la óptica hasta la ingeniería. Inclusive en el mundo natural que nos rodea, la polarización de la luz está presente. Por ejemplo, las abejas y otros insectos, aprovechan la luz polarizada del cielo para orientarse. Del mismo modo, varios sistemas ópticos contienen materiales birrefringentes, con índice de refracción dependiente de la polarización de la luz. Tal es el caso de las pantallas de cristal líquido LCD y los moduladores de luz, que se encuentran en ciertos visores 3D y utilizan birrefringencia inducida eléctricamente. La birrefringencia está presente también en muchos dispositivos médicos: por ejemplo, es usada en la medición del espesor del nervio óptico y en el diagnóstico del glaucoma. En general, varios materiales biológicos son birrefringentes y la 
microscopía hace uso de la luz polarizada en el análisis de tejidos. Además, la polarización es comúnmente usada en el estudio de la conformación de cristales y moléculas. En ingeniería mecánica una técnica basada en la birrefringencia ayuda a detectar estrés en modelos de estructuras y objetos.

Desafortunadamente, la polarización de la luz es usualmente considerada como un concepto secundario, especialmente en los programas de escuela superior. Con el propósito de llenar este vacío, se ha desarrollado un laboratorio relacionado con este tema, para la enseñanza en la educación secundaria y estudiantes en los primeros semestres de universidad. En este contexto los alumnos, con experimentos sencillos, tienen la posibilidad de entender la física de la polarización de la luz, sus conexiones con química y biología y los fundamentos de algunas de sus aplicaciones. Por ejemplo, cuando algunos materiales se sitúan entre dos filtros polarizadores cruzados, aparecen hermosas y fascinantes imágenes, que recuerdan operas de arte abstracto. Los estudiantes pueden crear sus propias composiciones artísticas, tomar fotos y realizar concursos artísticos entre ellos, sobre quién crea la obra más bella y original. Este es un ejemplo de cómo un arte figurativo, como la pintura o la fotografía, se puede vincular con la enseñanza de la física. De hecho, hay varios ejemplos en la literatura sobre la conexión entre física y música (PARKER, 2009); mientras la relación entre la física y las artes visuales casi siempre se centra exclusivamente en el tratamiento de la teoría del color (GUNTHER, 2012).

Otra razón por la que debería enseñarse la física de la luz polarizada, es que este concepto se usa frecuentemente para introducir la mecánica cuántica, tanto a nivel popular como a nivel de secundaria (GHIRARDI, 2007; MICHELINI; STEFANEL, 2021), así como en los primeros años universitarios (SAKURAI; NAPOLITANO, 2012; LVOVSKY, 2018).

Los autores del presente trabajo son conscientes de que, especialmente en América Latina, los cursos de física de las escuelas secundarias a menudo no logran introducir el electromagnetismo ni los fundamentos de la física moderna. Sin embargo, esta situación de retraso educativo respecto a otros países de Europa y Asia, (dependientes de muchos factores, sociales, económicos y políticos, que no analizáremos aquí), debe reducirse con todos los medios posibles. En lo que a nosotros respecta, y dentro de nuestro radio de acción, con este trabajo se espera hacer al menos una contribución dirigida a mejorar la preparación de los docentes, muchas veces procedentes de áreas distintas a la física, y con un conocimiento poco profundo de este tema.

En los siguientes párrafos se introducirán las propiedades fundamentales y las principales fuentes de luz polarizada, como el dicroísmo, la dispersión y la reflexión, y propiedades como la birrefringencia y actividad óptica, y algunos nuevos experimentos y visualizaciones de los fenómenos físicos involucrados, presentes en el laboratorio propuesto.

La secuencia de los párrafos, con sus contenidos, reproduce la secuencia didáctica que, a nuestro juicio, debería entrar en una introducción experimental y conceptual bastante completa a la polarización de la luz. El artículo finaliza con una introducción a la metodología 
del laboratorio propuesto y algunas sugerencias didácticas sobre su implementación en el aula.

\section{La polarización de la luz}

En este párrafo se resumen, para la conveniencia del lector, algunas propiedades fundamentales de las ondas electromagnéticas polarizadas, refiriéndose a la literatura para obtener más información (por ejemplo, se puede consultar el libro de Hecht (1991)).

Se puede imaginar un átomo, de manera simplificada, como una nube de carga negativa (los electrones), con un punto de carga masiva y positiva en su centro (protones y neutrones). Por la acción de un campo eléctrico, la carga positiva sufre un desplazamiento en el sentido del campo eléctrico y la carga negativa en el sentido opuesto (si la carga es $q$ y el campo eléctrico sobre la carga es $\vec{E}$, la fuerza generada en $q$ es $\vec{F}=q \vec{E}$ ). Por lo tanto, si hay un campo eléctrico oscilante armónico sobre el átomo, como el de una onda electromagnética de una frecuencia dada, podemos pensar en el átomo como un oscilador armónico forzado, que siente la fuerza del campo eléctrico $\vec{E}$ de la onda y la fuerza interna de Coulomb entre electrones y protones. Las cargas del átomo en condición de resonancia, vibran a la frecuencia de la onda electromagnética incidente y se comportan como un dipolo eléctrico oscilante. Los electrones acelerados, emiten ondas electromagnéticas y el dipolo eléctrico se convierte en una fuente de nuevas ondas electromagnéticas.

En la dirección ortogonal al dipolo, la onda electromagnética tiene la apariencia de la Fig. 1a: si el dipolo oscila en modo armónico y siempre en la misma dirección, el campo eléctrico de la onda emitida no cambia de dirección, y siempre oscila en un plano. Se dice entonces que la onda está polarizada linealmente. Además, si el dipolo eléctrico siempre oscila a la misma frecuencia, la onda electromagnética emitida, también tiene la misma frecuencia. Por ejemplo, en la Fig. 1a, tenemos una onda electromagnética polarizada a lo largo del eje z y que viaja a lo largo del eje x. Generalmente, la dirección de la polarización lineal está representada por un segmento que tiene dos flechas, y se omite la representación del campo magnético $\vec{B}$, que siempre es ortogonal al campo eléctrico $\vec{E}$ y deducible de éste. Además, el efecto del campo magnético de la onda electromagnética sobre la materia, es usualmente mucho menor que el efecto del campo eléctrico: del orden de $v / c$, donde $c$ es la rapidez de la luz, y $v$ la rapidez de las cargas sometidas al campo magnético. 

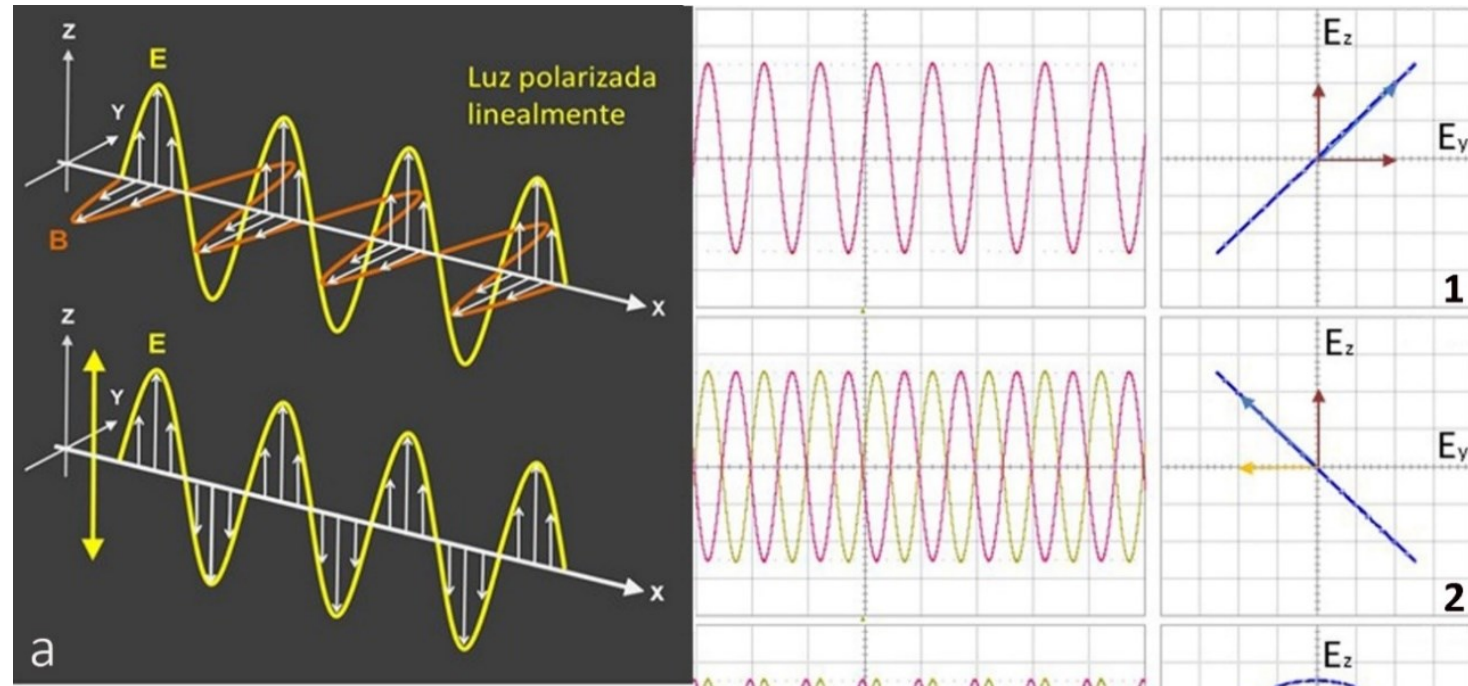

$E_{y}$
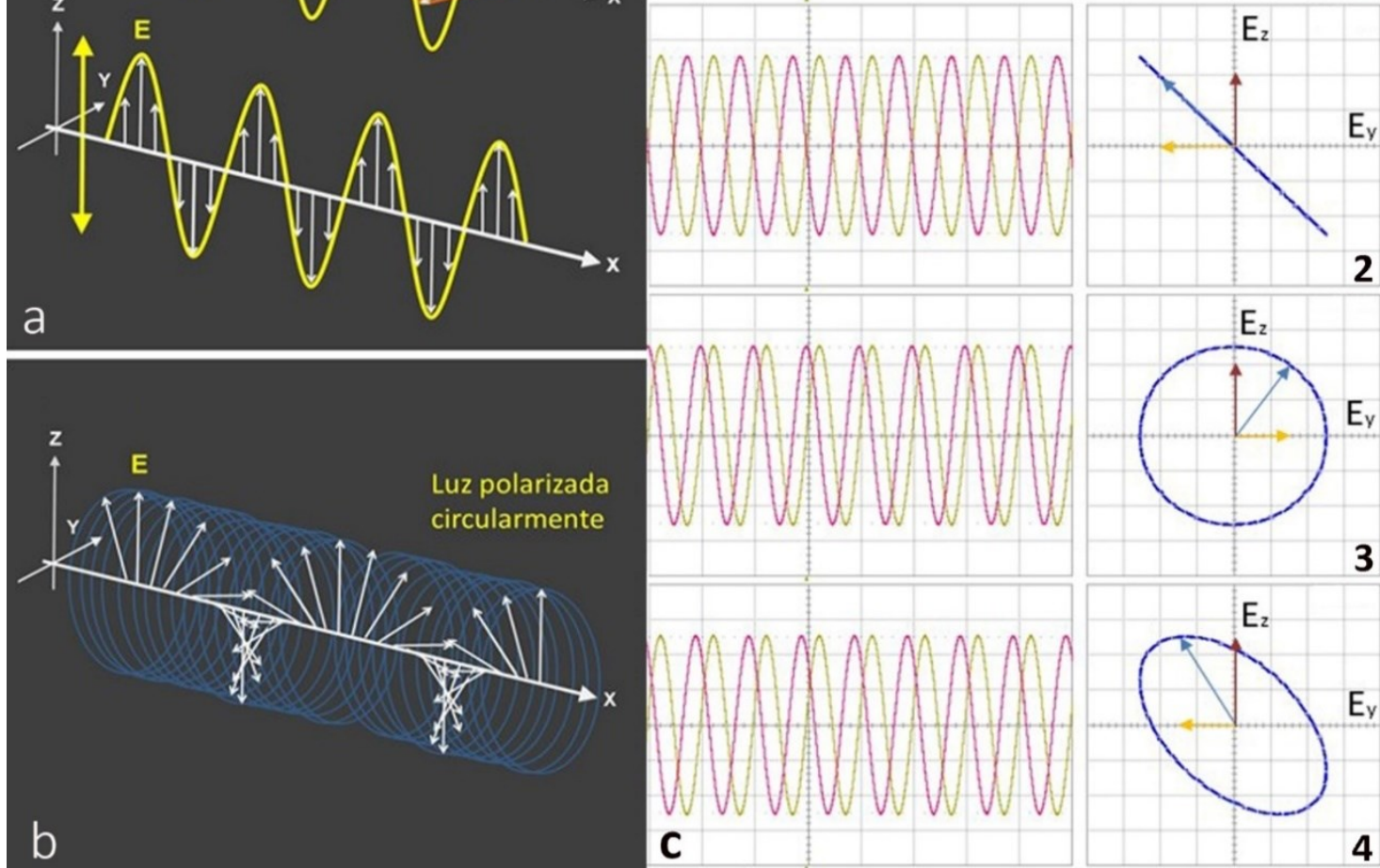

2
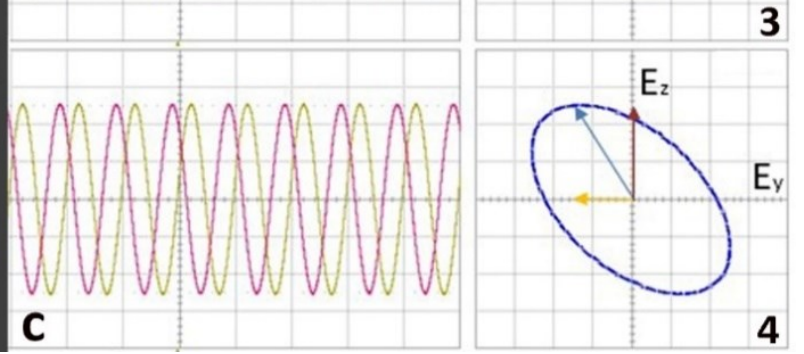

Fig. 1 -En a), se representa una onda electromagnética polarizada linealmente. La dirección de la polarización lineal, está representada por un segmento con dos flechas, donde la dirección del segmento es la del campo eléctrico. En b), se representa una onda electromagnética polarizada circularmente, donde los vectores del campo eléctrico rotan con velocidad angular constante y son de módulo constante. En cualquier punto, el campo eléctrico se puede imaginar como la suma de dos componentes oscilantes perpendiculares. Para la polarización lineal del campo eléctrico en c1) y c2), las dos oscilaciones están en fase o en contrafase. Si las oscilaciones componentes tienen un retraso de fase que corresponde a $\pi / 2$, la onda es polarizada circularmente. De forma general, para otros retrasos de fase, la onda es polarizada elípticamente, como en c4). Las gráficas en c), han sido obtenidas con un osciloscopio digital LeCroy 104MXs-A.

En general, el campo eléctrico $\vec{E}$ puede oscilar en cualquier dirección, y al ser un vector, puede representarse mediante la suma de dos vectores oscilantes perpendiculares (Fig. 1c). Para condiciones de desplazamiento de fase $\Delta \phi$ diferentes de las dos oscilaciones, y para diferentes amplitudes $E_{0 y}$ y $E_{0 z}$, el vector de campo eléctrico puede describir una recta, un círculo o un elipse (BETTINI, 2017). Sin embargo, la luz procedente del sol y de fuentes térmicas es principalmente no polarizada. De hecho, se debe pensar que la luz que llega a 
nuestros ojos es la suma de muchas ondas electromagnéticas, provenientes de diferentes átomos, cada uno emitiendo luz con polarización diferente. Además, la duración de las oscilaciones de los dipolos atómicos no es infinita, sino del orden del nanosegundo. En promedio, el resultado final observado es luz no polarizada, superposición de ondas con diferente frecuencia, donde el campo eléctrico cambia continuamente de dirección con el tiempo.

Para verificar si la luz está polarizada o para crear luz polarizada, se pueden emplear filtros polarizadores. En los experimentos realizados se han usado los filtros polarizadores del tipo inventado por Edwin Herbert Land en 1938. Land fue un científico americano, fundador de la Polaroid Corporation y también inventor de la fotografía instantánea.

En la tecnología óptica actual, los filtros polarizadores (polaroid) están constituidos de una hoja de capas con cadenas de polímeros de alcohol polivinílico. Las cadenas están alineadas y cubiertas con yodo, para hacerlas conductivas. De esta manera, una onda electromagnética puede inducir corrientes eléctricas a lo largo de los polímeros, y estas moléculas absorben la energía de la onda con la polarización en la dirección de las cadenas. Así, la luz se transmite principalmente en la dirección perpendicular a los polímeros (Fig. 2a). Esta dirección se llama dirección de polarización del filtro, o también eje de trasmisión.

Entonces, si un haz de luz no polarizada golpea un polarizador, la luz emergente desde allí es polarizada linealmente y perpendicularmente a las cadenas de alcohol polivinilo. Si se coloca un segundo filtro en el camino de este haz, como en Fig. 2b, con un ángulo de polarización de $90^{\circ}$ grados con respecto a la dirección de polarización del primer filtro, la luz se bloquea casi por completo (los mejores filtros pueden dejar pasar solo 1/100 de la intensidad de la luz). De esta forma, es posible verificar, por ejemplo, que la luz de la pantalla de una computadora portátil está polarizada linealmente, en dirección oblicua (Fig. 2c y 2d). Esta polarización corresponde a la dirección perpendicular al eje del polaroid en la posición que no permite el paso de la luz. En cambio, la transmisión de luz es máxima cuando los dos filtros se colocan con su dirección de polarización paralela (Fig. 3a).

En general, cuando la luz polarizada a lo largo de un cierto eje, pasa a través de un filtro con dirección de transmisión a un ángulo $\theta$ con respecto a este mismo eje, la intensidad $\mathrm{I}(\theta)$ de la luz transmitida es

$$
\mathrm{I}(\theta)=\mathrm{I}(0) \cos ^{2} \theta
$$

donde I(0) es la intensidad de la luz transmitida cuando es polarizada linealmente en la dirección de transmisión del filtro $(\theta=0)$. La relación anterior se llama ley de Malus, por el físico francés Étienne-Louis Malus, quien la formuló por primera vez en 1809. La propiedad que tienen ciertos materiales de absorber la luz en función de la dirección de polarización, se denomina dicroísmo. Esta palabra viene del griego dikhroos que significa dos colores. En general, se entiende por dicroísmo la propiedad que tienen algunos materiales de dividir un haz de luz incidente en diferentes colores. En el caso de la luz polarizada, la absorción de ésta por parte de un material, puede depender de la dirección de la polarización y también de la 
longitud de onda de la luz (entonces, por la luz visible, de su color). Sin embargo, para los filtros polaroid, el dicroísmo es prácticamente independiente de la longitud de onda.
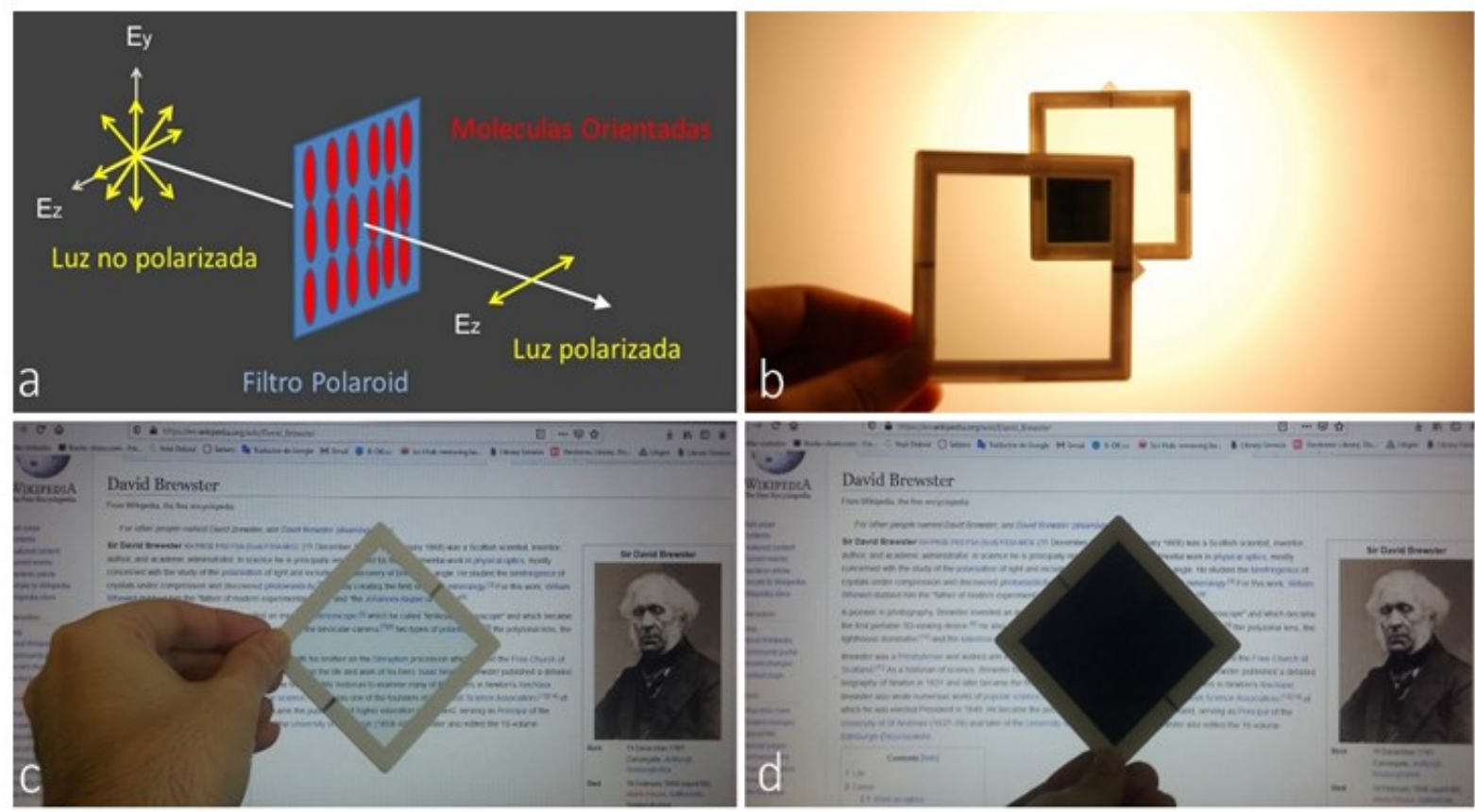

Fig. 2 - En a), una onda electromagnética no polarizada golpea un filtro polarizador. El campo eléctrico que pasa a través del filtro, tiene el único componente perpendicular a las moléculas y la luz saliente está polarizada linealmente en esta dirección. En b), una hoja blanca iluminada por detrás con una lámpara, emite luz no polarizada. Un polaroid polariza esta luz a lo largo de una dirección determinada. Un segundo filtro, con eje de transmisión ortogonal a esta polarización, bloquea completamente la luz. En c) y d), al girar un filtro polarizador frente a la pantalla del portátil, se puede verificar la polarización de la luz emitida por ésta.

Para ilustrar el comportamiento de los filtros polarizadores, los estudiantes pueden realizar el siguiente experimento. Si una hoja blanca se ilumina por detrás con una lámpara, ésta emite luz no polarizada. Un polaroid polariza esta luz a lo largo de una dirección determinada, como se puede mirar observando la luz a través de un segundo filtro (Fig. 2b). Como alternativa, se puede usar la luz emitida por un puntero láser, que, de manera usual, se encuentra parcialmente polarizada, así como en los experimentos descritos en Fig. 3. Si los filtros tienen eje de transmisión paralelo, la luz pasa casi completamente a través del segundo filtro (Fig. 3a). Sin embargo, si los dos filtros están perpendiculares, la luz está casi completamente bloqueada por el segundo polaroid (Fig. 3b). Un tercer filtro intermedio puede hacer reaparecer "mágicamente" la luz, si crea un componente del campo eléctrico con polarización no ortogonal a la dirección de transmisión del último polaroid (Fig. 3c y 3d). 

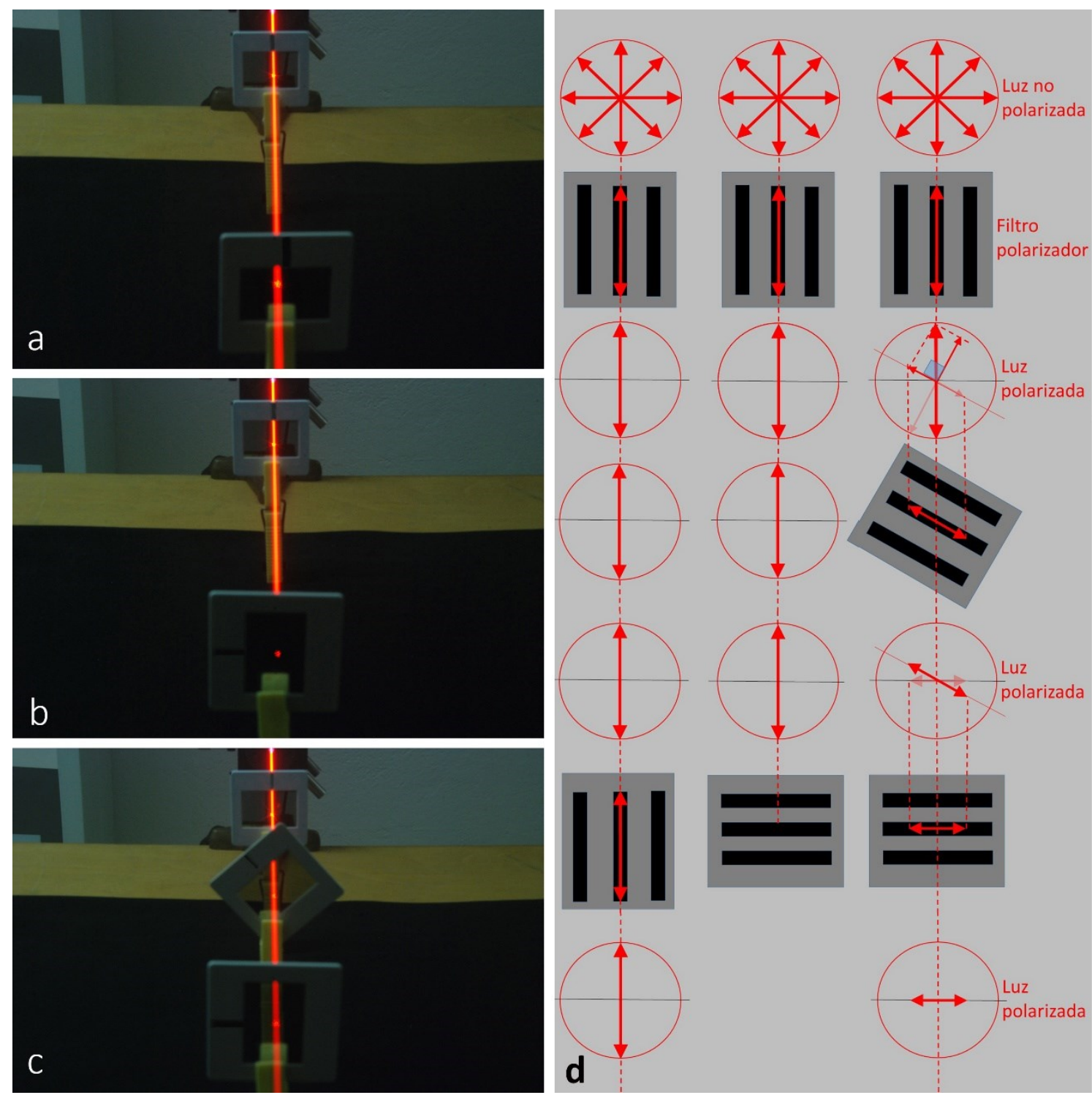

d

Fig. 3 - En a), b), y c), un primer filtro, polariza verticalmente la luz emitida por un puntero láser. Si los filtros tienen eje de transmisión paralelo, como en a), la luz pasa casi completamente a través de ellos; pero, si los dos filtros están perpendiculares, la luz está casi completamente bloqueada por el secundo polaroid (b). Un tercer filtro intermedio puede dejar pasar la luz después del último filtro, si crea un componente del campo eléctrico con polarización no ortogonal a la dirección de transmisión de éste, como se muestra en c). En d) se reproduce esquemáticamente la situación que ocurre en los tres experimentos.

Los filtros polarizadores se usan a menudo en fotografía, gafas de sol, para minimizar la luz reflejada desde las superficies (que como se observará en el párrafo IV, está polarizada en una dirección privilegiada) y ahora, también en algunos dispositivos de visualización 3D. Los polarizadores también se utilizan en varios sistemas de comunicación óptica y en las pantallas LCD de las computadoras. 


\section{Las abejas bailantes de Karl Von Frisch}

Según lo observado por primera vez por el físico francés Dominique F. J. Aragó en 1809 , la luz del cielo es polarizada: el grado de polarización es muy alto a $90^{\circ}$ grados con respecto a la dirección de la luz solar y disminuye tanto hacia adelante como hacia atrás de este ángulo. Es posible dar una explicación cualitativa simple del origen de este fenómeno siguiendo la demostración de Lord Rayleigh (ALONSO; FINN, 1970).

Considere, cómo se describió anteriormente, un átomo o molécula como un pequeño dipolo eléctrico (sin embargo, el siguiente razonamiento se aplica también a partículas de humo o polvo que tienen pequeñas dimensiones en comparación con la longitud de onda de la luz). Cuando la luz incidente sobre el dipolo es polarizada linealmente, el dipolo eléctrico empieza a oscilar en la dirección del campo eléctrico de la onda, es decir, en la dirección de la polarización. Entonces, a su vez, el dipolo oscilante, emite la energía absorbida de la onda incidente en forma de radiación electromagnética y dispersa la luz. La luz dispersa tiene la polarización del haz incidente y una distribución de la intensidad característica, en particular, no hay luz emitida en la dirección de oscilación del dipolo (Fig. 4a-c).

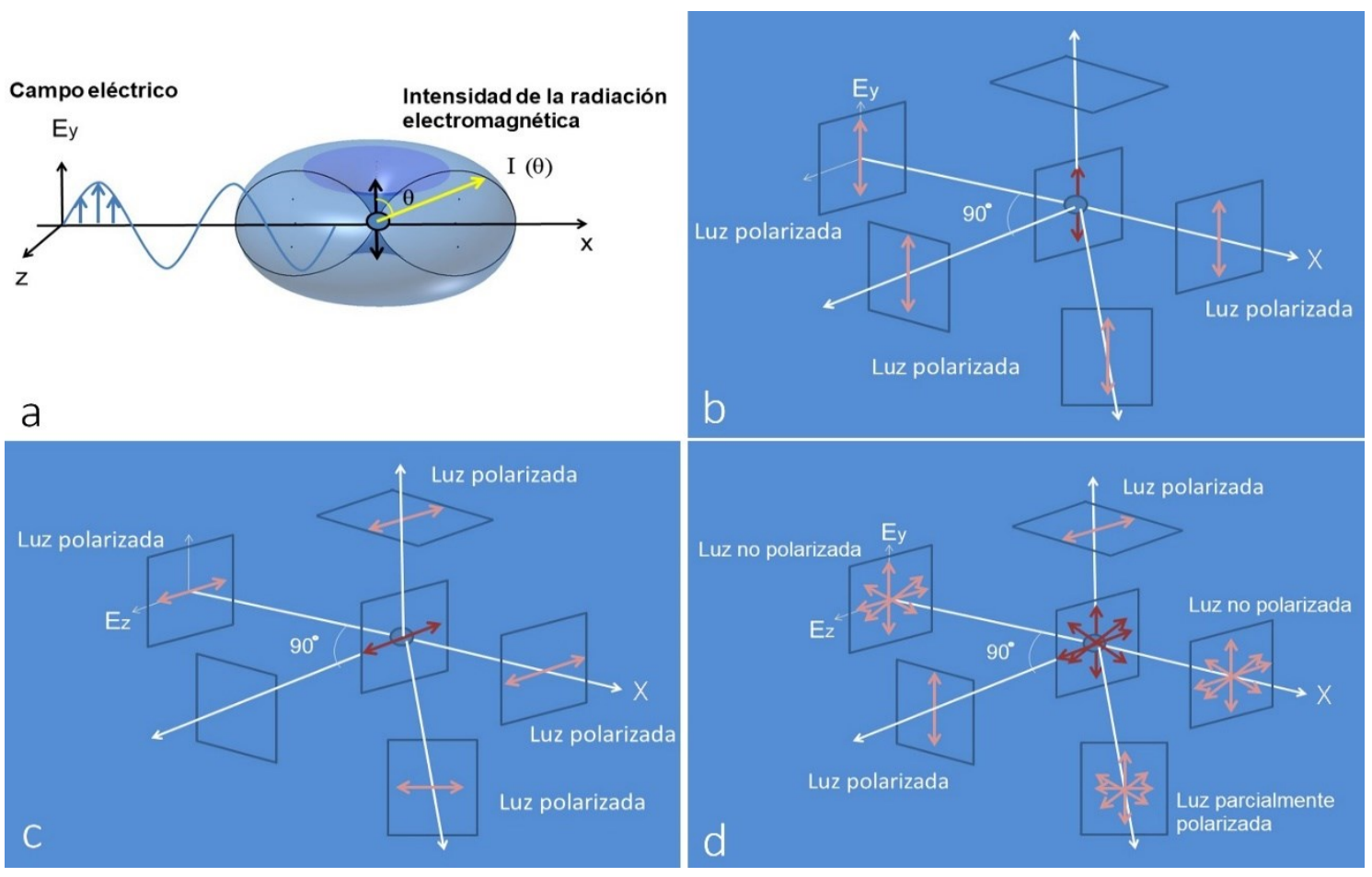

Fig. 4 - a) El campo eléctrico de una onda incidente en un átomo, crea un dipolo eléctrico oscilante, que a su vez emite radiación. La intensidad de esta radiación es mínima en la dirección de oscilación del dipolo, y máxima en el plano ortogonal al dipolo. b), c) y d) El dipolo siempre oscila transversalmente a la dirección de propagación de la onda electromagnética incidente. Por lo tanto, mirando en una dirección ortogonal, hacia el rayo, la luz difundida por el dipolo está polarizada. 
Sin embargo, incluso si la radiación incidente no está polarizada, la radiación dispersa puede ser polarizada o parcialmente polarizada. De hecho, las oscilaciones inducidas en las cargas eléctricas del dipolo, están siempre en un plano perpendicular a la radiación incidente. Entonces, la radiación dispersa paralela a este plano, está linealmente polarizada, como se puede comprender al observar la Fig. 4d.

Es posible estudiar fácilmente este efecto de polarización por dispersión en laboratorio, reproduciendo los experimentos de John Tyndall (TYNDALL, 1882). El físico irlandés, en la década de 1860, mostró que pequeñas partículas de polvo, de humo fino, o pequeñas gotas de agua, dispersan la luz de longitud de onda $\lambda$ más corta, mucho más que la de $\lambda$ más grande. Por ejemplo, esta es la razón del color azul del humo. En el caso de las moléculas de aire, este efecto explica el color azul del cielo. Tyndall examinó la luz que se dispersa por el humo dentro de un largo tubo de vidrio y descubrió que esta se polarizaba en la dirección normal al haz incidente. Se puede recrear este experimento con agua y un poco de leche para aumentar la luz dispersa (en este caso las moléculas de la grasa de la leche realizan la mayor parte del trabajo). En las imágenes de la Fig. 5, se usa un acuario lleno de agua y el haz luminoso de un viejo proyector por tobogán. De esta manera, es posible comprobar, experimentalmente, que sólo la luz con polarización ortogonal con respecto a la dirección del haz está dispersa transversalmente al haz de luz (véase también la Fig. 6a.).

La polarización de la luz puede ser mejor visualizada si se construye un polariscopio, es decir, un dispositivo para detectar la polarización. El polariscopio más simple, está formado por dos piezas de filtros polarizadores con direcciones de polarización en ángulos rectos entre sí, como se muestra en la Fig. 6b. Se puede usar el contraste de brillo entre los dos filtros, como detector de polarización más sensible que usando un solo polaroid.
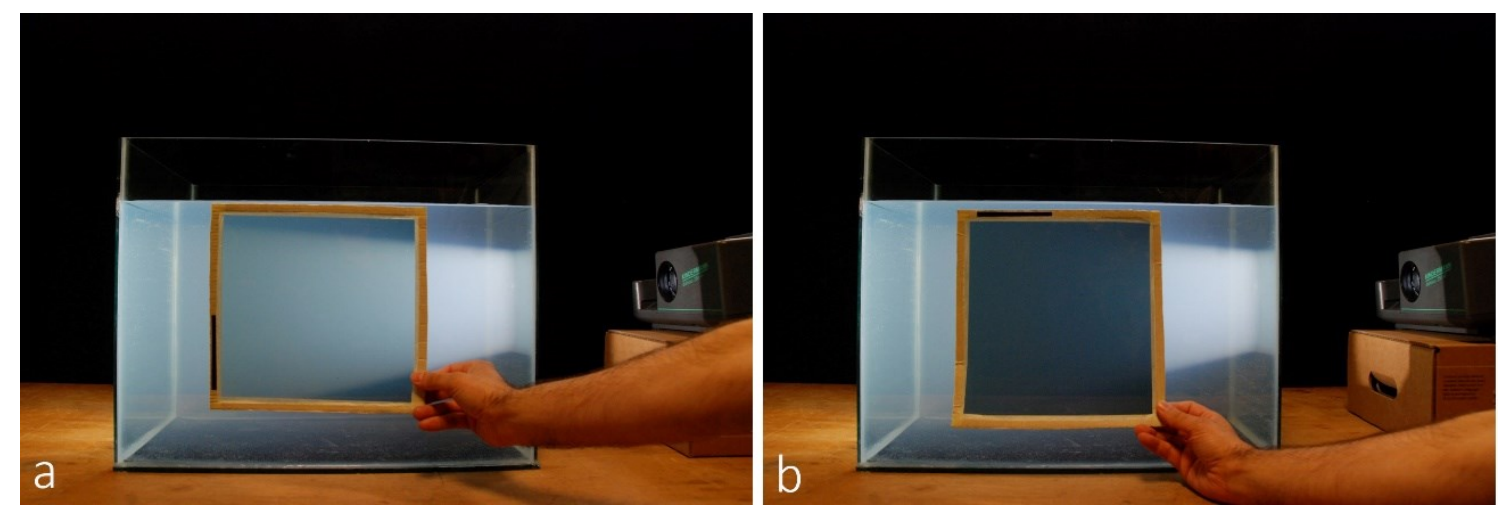

Fig. 5 - El experimento de Tyndall. En a), se observa cómo la luz dispersa tiene polarización ortogonal con respecto a la dirección del haz. En b), en cambio, se puede verificar cómo la luz con polarización paralela con respecto al haz, prácticamente no se dispersa (la línea negra en el filtro indica la dirección de transmisión del polaroid). 

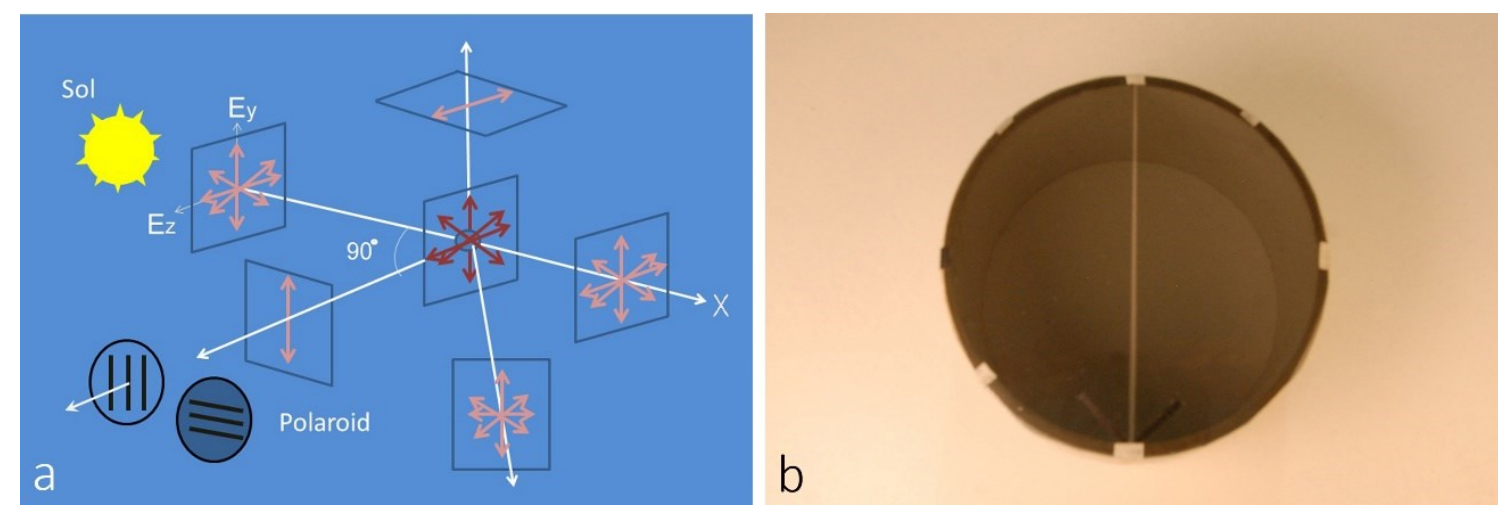

Fig. 6 - a) Mirando en el plano de oscilación del dipolo, la luz dispersa del dipolo está polarizada. Esto significa que la dirección de transmisión de un filtro polaroid que se ve oscuro, da la dirección del rayo y la dirección de la posición del sol. En b), se reproduce un polariscopio hecho de dos piezas de polaroid colocadas una cerca de la otra, con ejes de transmisión ortogonales (indicados por las dos pequeñas líneas negras).

Con un polariscopio, la polarización de la luz del cielo es más evidente, tal como se puede verificar en la Fig. 7. Según la explicación para la polarización de la luz por dispersión, la ubicación del sol se encuentra observando el pedazo de filtro más obscuro del polariscopio, que no deja pasar la radiación del cielo polarizada. La dirección de polarización de este filtro, da la dirección donde es posible encontrar el sol (Fig. 6a y 7b).

Un tipo diferente de polariscopio fue inventado por Karl von Frisch, científico austríaco, famoso por sus estudios sobre la fisiología de las abejas, su comportamiento y el significado de su danza (VON FRISCH, 1966). Von Frisch observó que al colocar un filtro polarizador entre el sol y las abejas, estas modificaban su conducta. De hecho, resulta que las abejas de miel (Apis mellifera) en la colmena se mueven realizando una especie de baile, formando una figura en forma de ocho (Fig. 8a). De esta manera ellas comparten información sobre la localización de los alimentos con sus compañeras. Según el código de comunicación de las abejas, descubierto por von Frisch, la dirección vertical indica la dirección del sol, el ángulo del ocho, con respecto a la vertical, muestra la dirección donde está el alimento con respecto al sol y la colmena, mientras que la duración de la danza es una medida de la distancia entre el alimento y la colmena.

Las abejas de miel, como muchos artrópodos, poseen dos ojos compuestos. Éstos están constituidos por unidades repetidas llamadas omatidios: en un ojo de abeja, hay alrededor de 5000 de estas estructuras. Cada omatidio es un sistema visual independiente: tiene una lente, un cono cristalino transparente y células de pigmentos que separan un omatidio de los otros. También hay células visuales dispuestas como la sección de una naranja, formando la retinula, una estructura correspondiente de la retina humana. Según von Frisch, las ocho células de la retinula son sensibles a cuatro diferentes direcciones de polarización (Fig. 8c). 


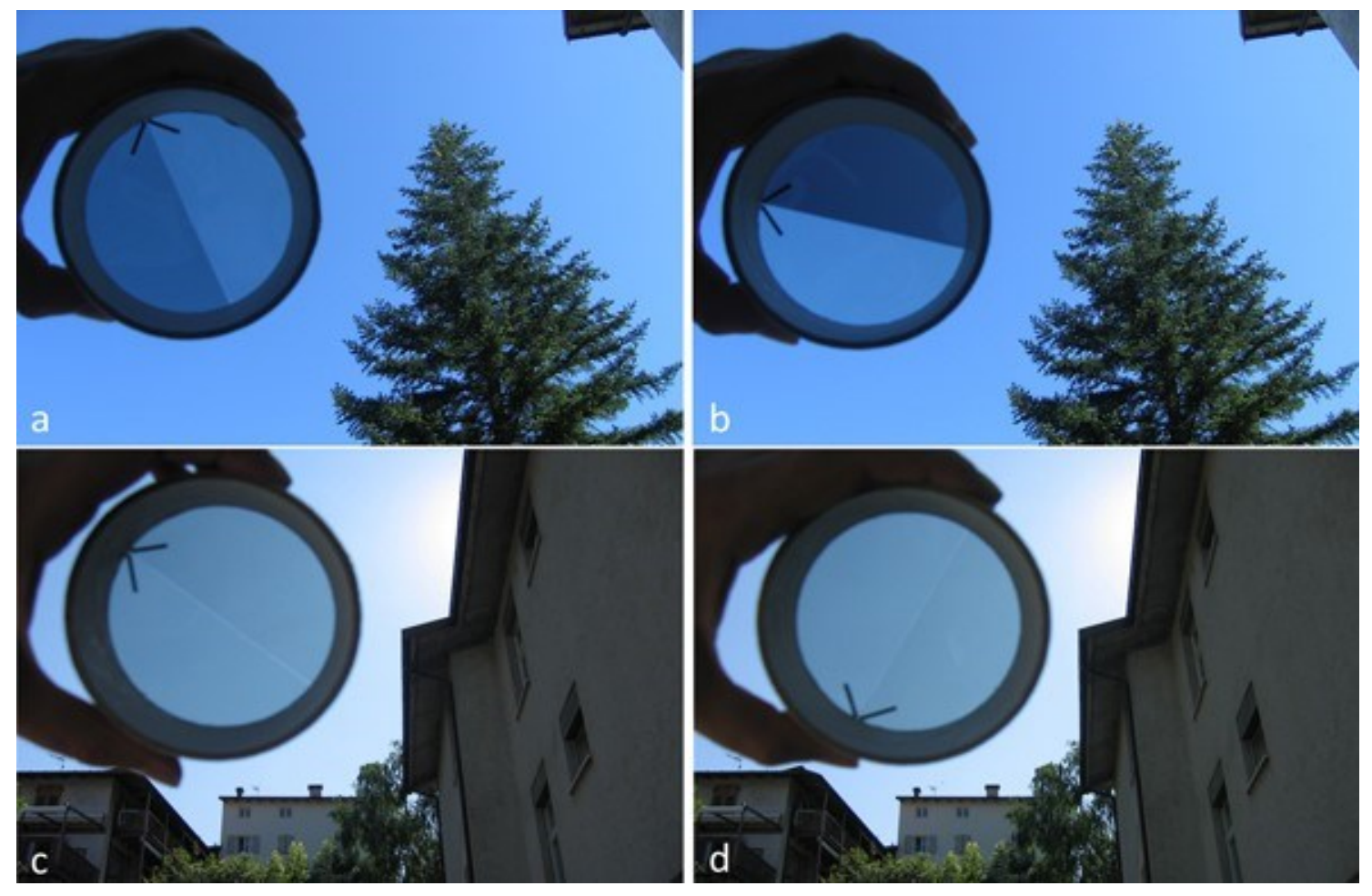

Fig. 7 - a) y b) A $90^{\circ}$ grados con respecto a los rayos del sol, la luz resulta bastante polarizada, y la dirección de polarización del filtro más obscuro, nos da la dirección donde es posible encontrar el sol. Sin embargo, mirando al sol, la luz está muy poco polarizada, como se observa en c) $y d$ ).

Con este sistema visual, las abejas son capaces de detectar de manera eficiente la posición del sol, incluso en un día nublado. El científico austríaco elaboró un modelo de la retinula de la abeja, usando piezas de un filtro polaroid, que funciona como un polariscopio. Sin embargo, las cosas pueden ser diferentes: según Pye, en la retinula cuatro células son sensibles a una dirección de polarización y las otras cuatro a la dirección perpendicular a ésta, tal como en la Fig. 8d; además existe una célula central (PYE, 2001).

Por lo tanto, con los estudiantes se ha elaborado el mismo polariscopio de von Frisch (foto a la izquierda en la Fig. 8b) y otro modelo de retinula, tal vez más apegada a su estructura real, con sólo dos diferentes direcciones de polarizaciones, tal como se puede observar en la imagen de la derecha de la Fig. 8b. Se pueden probar los polariscopios propuestos, por ejemplo, detectando la polarización de la pantalla del ordenador portátil o del cielo (Fig. 9).

Las abejas no son los únicos insectos que pueden detectar y utilizar la polarización de la luz. Hay estudios que muestran que ciertas hormigas del desierto como la Cataglyphis fortis también usan la luz polarizada del cielo para orientarse. Los notonéctidos (del género Notonecta) y otros insectos acuáticos, por otro lado, usan su habilidad de revelar la luz polarizada por reflexión para encontrar charcos de agua (JOHNSEN, 2011). 

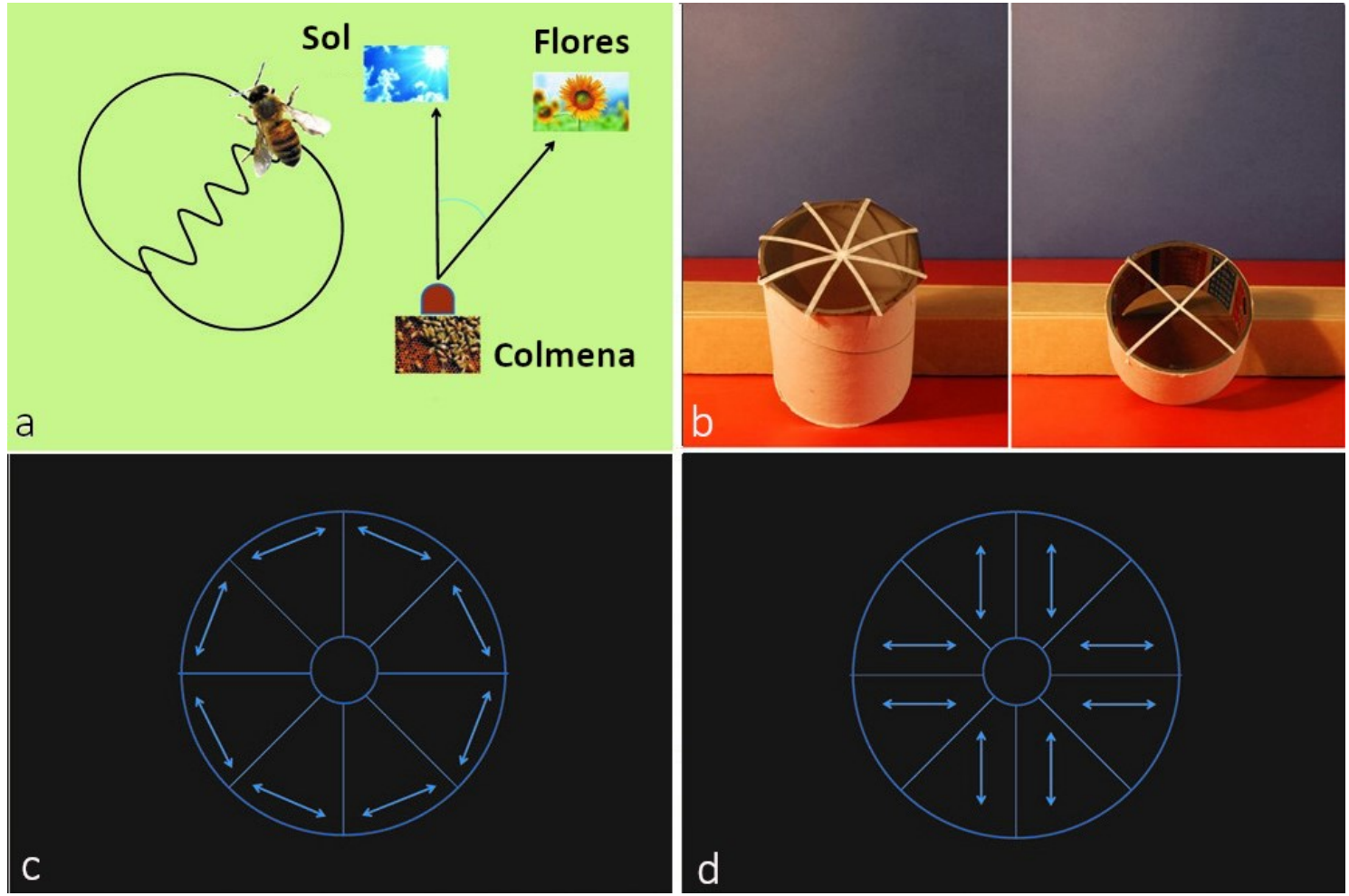

Fig. 8 - a) La abeja, para comunicar a sus compañeras el lugar en el cual se encuentra la comida, describe una figura en forma de ocho. La dirección vertical en la colmena indica el sol y la dirección de la parte central del ocho indica la dirección donde se encuentra la comida. En b), hay dos tipos de polariscopios construidos, respectivamente, siguiendo a von Frisch (izquierda) y Pye (derecha). En d) y c), se dibujan las disposiciones de las piezas de polaroid de los dos polariscopios, con sus respectivos ejes de transmisión.

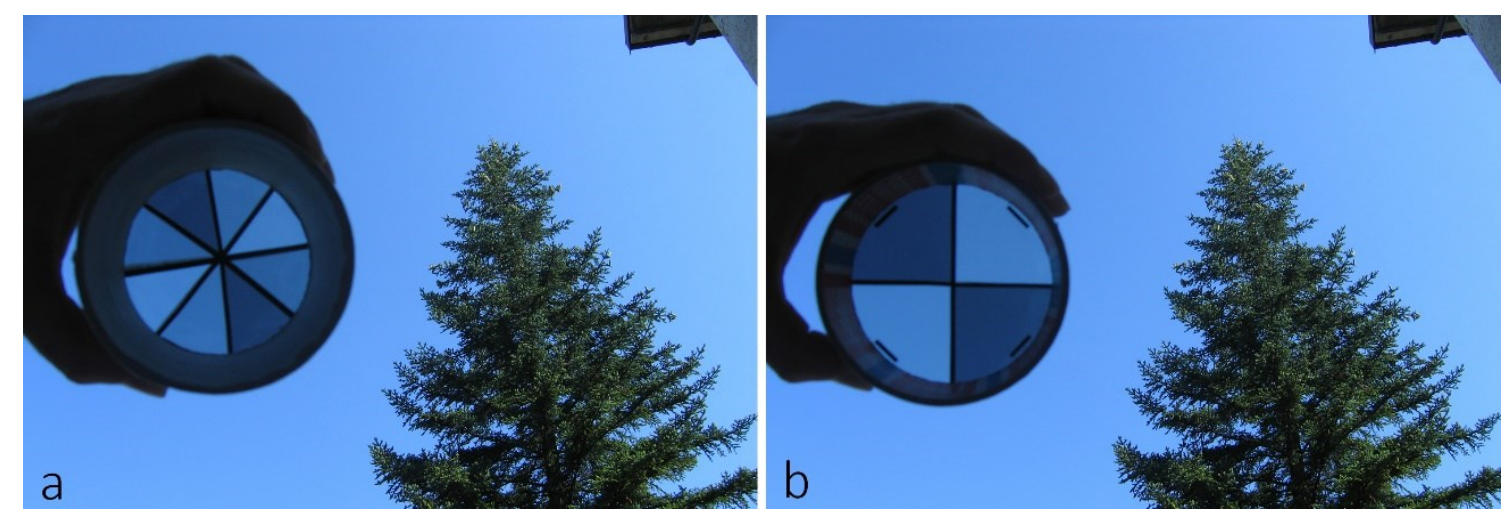

Fig. 9 - a) A la izquierda se tiene el polariscopio ideado por von Frisch para reproducir la visión de la polarización de la luz del cielo de las abejas. En b), se representa otra versión de polariscopio con solo dos direcciones diferentes de los filtros polarizadores. Las pequeñas líneas negras indican los ejes de transmisión. 
Muchos colores iridiscentes de los insectos, especialmente las mariposas, son producidos por la reflexión y la interferencia de la luz, y por lo cual se ven diferentes a través de un filtro polarizador. La investigación sobre las mariposas de la especie Heliconius, ha revelado que éstas usan la luz polarizada reflejada por sus alas para reconocerse entre ellas. Si hay vertebrados que pueden revelar la polarización de la luz, es un hecho mucho más controvertido, así como el uso que se le podría dar a esta habilidad. Tal vez hay peces que usan esta facultad para mejorar su visión en el agua, o para reconocer a otras especies (JOHNSEN, 2011).

\section{Reflejos mágicos}

Cuando la luz encuentra una superficie límite entre dos materiales con diferentes índices de refracción, ésta en parte se refleja y en parte se transmite. Como descubrió Malus, la fracción de luz reflejada, depende de la polarización del rayo entrante y del ángulo de incidencia que éste forma con la dirección ortogonal a la superficie.
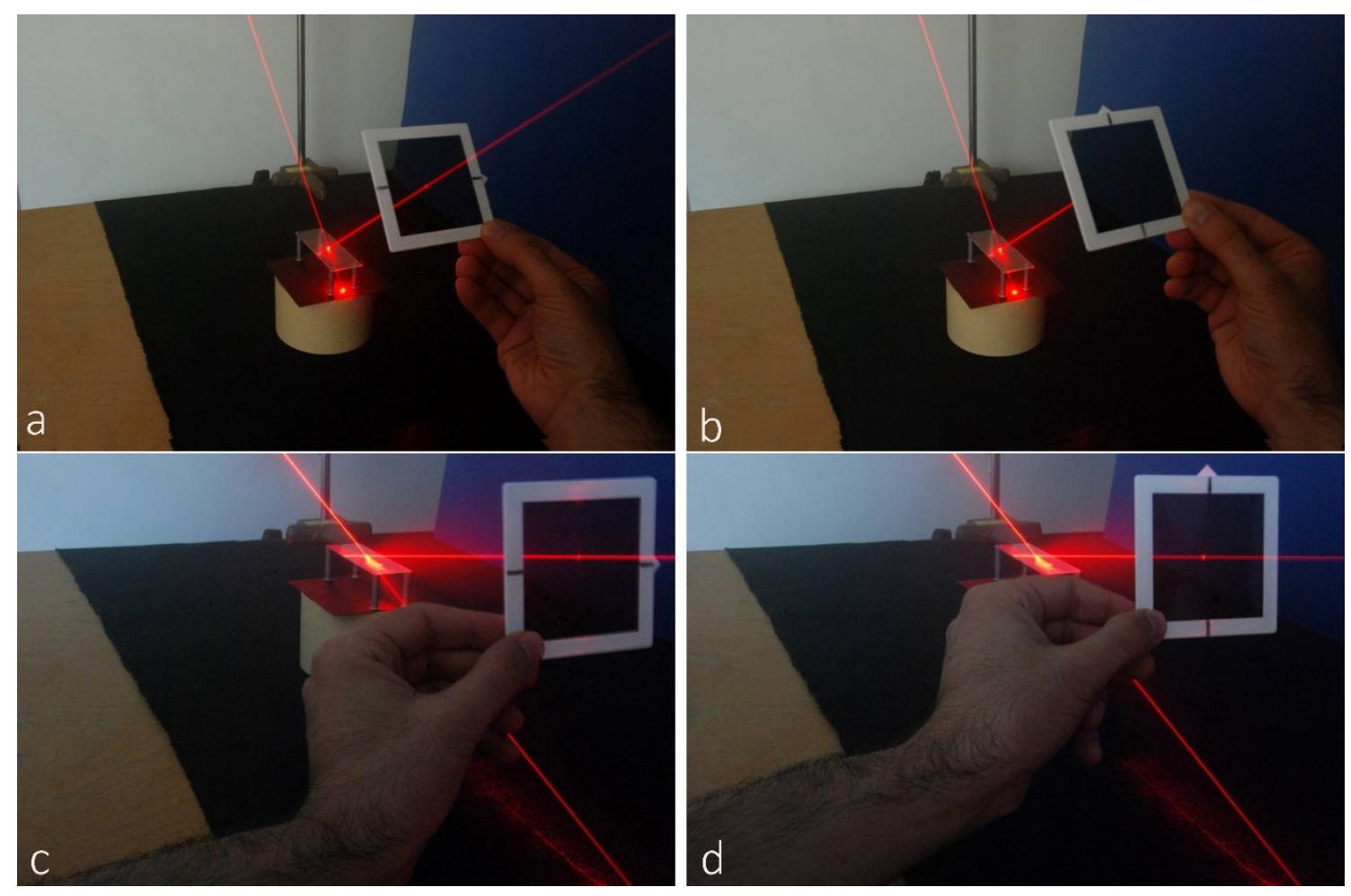

Fig. $10-a)$ y b) Un rayo láser no polarizado se refleja en un portaobjetos de vidrio por microscopio. En un ángulo particular, el rayo reflejado está prácticamente polarizado, con polarización paralela a la superficie reflectante. Para diferentes ángulos, la luz reflejada está solo parcialmente polarizada, como se puede apreciar en las fotos c) yd).

Además, cuando una luz no polarizada incide con un ángulo particular, llamado 
ángulo de polarización o ángulo de Brewster, la luz reflejada desde la superficie está casi de forma completa linealmente polarizada y la polarización es paralela a la superficie reflectante (Fig. 10a, b); para ángulos diferentes del ángulo de Brewster, la luz reflejada está solo parcialmente polarizada (Fig. 10c, d), (ALONSO; FINN, 1970). Se puede dar una explicación simple sobre la existencia de la polarización por reflexión, imaginando los átomos de los materiales como dipolos oscilantes, de manera similar a lo descrito en el párrafo III.

El campo eléctrico del rayo de luz incidente, que penetra en el material, hace oscilar las cargas de los átomos del mismo, que reemiten ondas electromagnéticas. Estas ondas electromagnéticas, sumadas a las ondas del rayo incidente, crean el rayo refractado. El rayo reflejado se debe principalmente a las ondas emitidas por las capas de átomos superficiales del material reflectante. Las cargas del material oscilan en un plano perpendicular al rayo refractado. Por lo tanto, si el rayo reflejo está en este plano, y entonces es ortogonal al rayo refractado (Fig. 11a), la luz reflejada está linealmente polarizada paralela a la superficie reflectante, como se ha observado en el efecto ya explicado en la Fig. 6 a.

Si denotamos el ángulo de incidencia con $\theta_{\mathrm{i}}$, y con $\theta_{\mathrm{r}}$ el ángulo de refracción, la luz reflejada se polariza al máximo valor cuando es válida la relación (Fig. 11a):

$$
\theta_{i}+\theta_{r}=180^{\circ}-90^{\circ}=90^{\circ}
$$

es decir:

$$
\theta_{r}=90^{\circ}-\theta_{i}
$$

Desde la ley de Snell, se conoce que:

$$
\frac{\sin \theta_{i}}{\sin \theta_{r}}=\frac{n_{2}}{n_{1}}
$$

donde $n_{2}$ y $n_{1}$ son, respectivamente, el índice de refracción del material reflectante y del medio por donde viaja el rayo incidente. Remplazando la Ec. (3) en la Ec. (4), y sustituyendo $\theta_{i}=\theta_{B}$, se obtiene:

$$
\theta_{B}=\arctan \left(n_{2} / n_{1}\right) \text {. }
$$

El ángulo de incidencia particular $\theta_{B}$ que satisface la relación anterior, se llama ángulo de Brewster, o ángulo de polarización, y es el ángulo que corresponde a la máxima polarización por reflexión. El ángulo de polarización es cercano a $53^{\circ}$, para una superficie de agua en aire y a $56^{\circ}$, para una superficie de vidrio en aire. En 1808, Malus ya había descubierto que la luz reflejada estaba parcialmente polarizada. Sin embargo, la relación cuantitativa se debe al físico escocés David Brewster, quien en 1812 descubrió la ley que lleva su nombre. Los inventos del caleidoscopio y el estereoscopio también se deben a Brewster. 

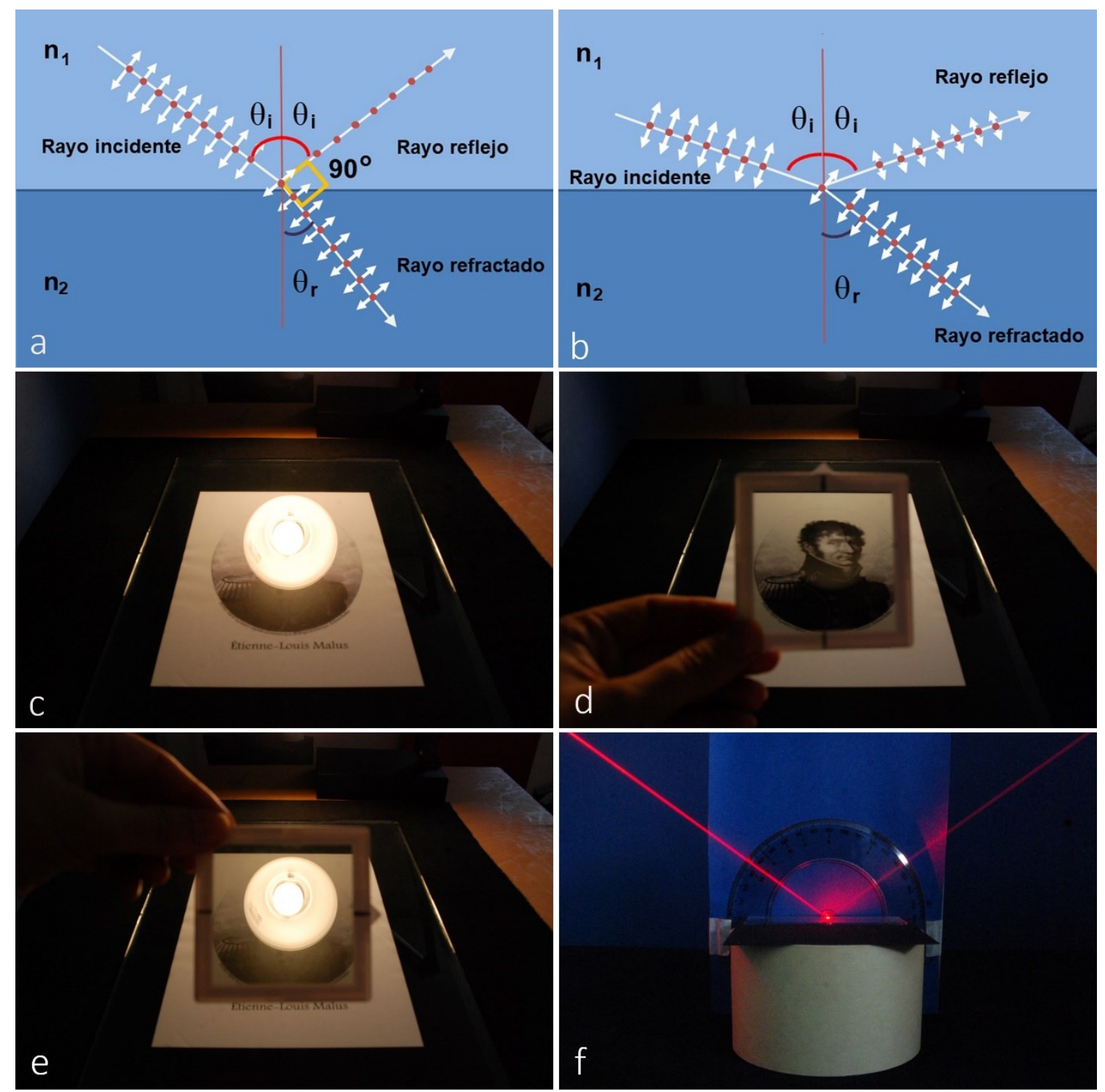

Fig. $11-a)$ Las cargas del material oscilan ortogonalmente al rayo refractado, y cerca de la superficie de separación, producen el rayo reflejado. La radiación reflejada está polarizada, si el rayo reflejado pertenece al plano de oscilación de las cargas. La polarización será paralela a la superficie reflectante (los vectores de polarización, en el dibujo ortogonales a la página, están indicados con puntos). b) Si el rayo reflejado no pertenece al plano de oscilación de las cargas, la radiación reflejada puede tener un componente de la polarización en el plano ortogonal a la superficie de separación. c) Es posible eliminar o disminuir el reflejo que dificulta la visión de una imagen, a través de un polaroid con un eje de transmisión ortogonal a la polarización de la luz reflejada (d)), como se puede verificar, rotando el polaroid (e)). f) Al colocar un goniómetro cerca del rayo de incidencia, se puede dar una estimación del ángulo de reflexión en las diversas situaciones experimentales. 
En la Fig. 11c, se tiene el retrato de Malus debajo de una superficie de vidrio, con el reflejo de una lámpara. La intensa reflexión, no permite distinguir la imagen de Malus. Sin embargo, si el ángulo de reflexión está cerca del ángulo de Brewster, es posible eliminar casi por completo el reflejo (Fig. 11d) con un filtro que tenga un eje de transmisión ortogonal a la polarización de la luz reflejada, haciendo visible la imagen debajo del vidrio. De hecho, dado que la imagen de Malus, que se ve a través de la luz de la lámpara, absorbida y difundida por el papel, es de luz no polarizada, una parte de su luz puede pasar el filtro y alcanzar los ojos del espectador. Por el contrario, el filtro bloquea el reflejo de la luz polarizada horizontalmente (Fig. 11e). Las gafas de sol con filtros polarizadores funcionan de acuerdo con los principios físicos que se acaba de describir: ellas minimizan los reflejos de luz polarizada que pueden dificultar o molestar la visión.

En la Fig. 11f, por medio de un goniómetro, se muestra la manera de estimar el ángulo de Brewster, variando el ángulo de incidencia y observando cuando la luz, transmitida con polarización en el plano que contiene el haz reflejado y ortogonal a la superficie, es mínima.

\section{Arte con basura}

El celofán es un polímero compuesto de largas cadenas de moléculas alineadas de glucosa. Las ondas electromagnéticas con polarización ortogonal a las moléculas, viajan a través de este material a diferente velocidad, con respecto a las ondas con polarización paralela a las cadenas. Por lo tanto, este material se comporta como si tuviera diferentes índices de refracción, en función de las dos direcciones ortogonales de la polarización de la luz. Varios materiales tienen un comportamiento similar al celofán, por ejemplo, los cristales con estructura atómica y molecular asimétrica, como la calcita, el cuarzo o la turmalina. Esta propiedad es llamada birrefringencia (ALONSO; FINN, 1970).

Se puede entender cualitativamente este fenómeno, imaginando una vez más a los átomos, o las moléculas del medio donde viaja la onda electromagnética, como dipolos eléctricos. Cuando el campo eléctrico de la onda se propaga en el medio, induce dipolos eléctricos oscilantes, que a su vez emiten ondas. La suma de estas ondas con la onda inicial, da la onda refractada, con una velocidad de fase $v$ disminuida por un factor dado por el índice de refracción $n: v=c / n$. Sin embargo, todo esto es correcto si el medio es isotrópico, es decir, si tiene las mismas propiedades en todas las direcciones. Considere, por ejemplo, una molécula biatómica como una nube de carga negativa alrededor de dos centros de carga positiva. En este caso, las oscilaciones inducidas por el campo eléctrico serán diferentes, si el campo eléctrico de la onda es ortogonal o paralelo a la línea geométrica que une los dos núcleos positivos: para el campo eléctrico, la molécula no es un sistema isotrópico (BETTINI, 2017). Esto sucede en la mayoría de las moléculas. Sin embargo, cuando la radiación electromagnética se propaga en un sistema como un gas, un líquido o un sólido amorfo, 
compuesto de muchas moléculas orientadas al azar en el espacio, como un efecto promedio, el sistema se comporta como isotrópico.

Existen algunos cristales donde las moléculas o los átomos forman retículos cúbicos, que se comportan como materiales isotrópicos. Pero también, hay otros tipos de cristales con estructura diferente. Por ejemplo, la estructura de la calcita tiene un eje particular, llamado eje óptico (Fig. 12a), de modo que, si el campo eléctrico de la onda tiene una polarización ortogonal a este eje, el medio actúa como si fuera isotrópico. Sin embargo, si la polarización tiene una componente paralela a este eje, el cristal se comporta como no isotrópico.

Siguiendo la primera explicación desarrollada por Christiaan Huygens en 1690 de la birrefringencia, se puede pensar que, si el campo eléctrico de la onda incidente es perpendicular al eje óptico, las ondas secundarias que se crean en el material, según el principio de Huygens (YÁNEZ, 2018), viajan con la misma velocidad en todas las direcciones. El frente de estas ondas es esférico, y la superposición de estas da lugar al rayo refractado, que sigue la ley de refracción de Snell, como en un material isotrópico.

Si en cambio, la polarización no es perpendicular a este eje, el medio no se comporta como isotrópico. Las ondas de Huygens viajan con diferentes velocidades en diferentes direcciones, y sus frentes de onda no son esféricos, sino que, como puede demostrarse, son elipsoidales (ALONZO; FINN, 1970). La suma de estos frentes crea un rayo refractado que no sigue la ley de Snell; por esta razón, este rayo se nombra extraordinario. Al contrario, el rayo que sigue la ley habitual de refracción se llama ordinario (Fig. 12a, b).

En el párrafo II, se observó que una onda electromagnética se puede imaginar como la suma de dos ondas con polarizaciones ortogonales. Cuando estas dos ondas componentes viajan en un material birrefringente, se propagan en el medio con diferente velocidad y diferentes índices de refracción. Entonces, en un cristal birrefringente, un rayo de luz incidente no polarizado se separa en dos haces: el rayo ordinario y el rayo extraordinario, cada uno con polarización lineal y ortogonal entre ellos. Un rayo es asociado a un mayor índice de refracción y tiene velocidad de fase más baja con respecto a otro, con índice de refracción más bajo y velocidad de fase más alta.

Si el material birrefringente es delgado, como el celofán, no existe una separación detectable del rayo incidente en dos partes. Cuando el rayo sale del material, los dos componentes de polarización ortogonal se suman, para dar una única onda electromagnética resultante. Sin embargo, en general, esta onda tendrá una polarización diferente de la radiación incidente. Esto se debe a que la onda componente más lenta es retrasada con respecto a la otra, y este retraso da como resultado una diferencia de fase entre las oscilaciones componentes. Esto significa que, en función del retardo de fase, la onda resultante saliente puede tener una polarización lineal rotada, o por lo general, polarizarse circularmente o elípticamente, en comparación con una onda inicial polarizada linealmente (Fig. 12c). 

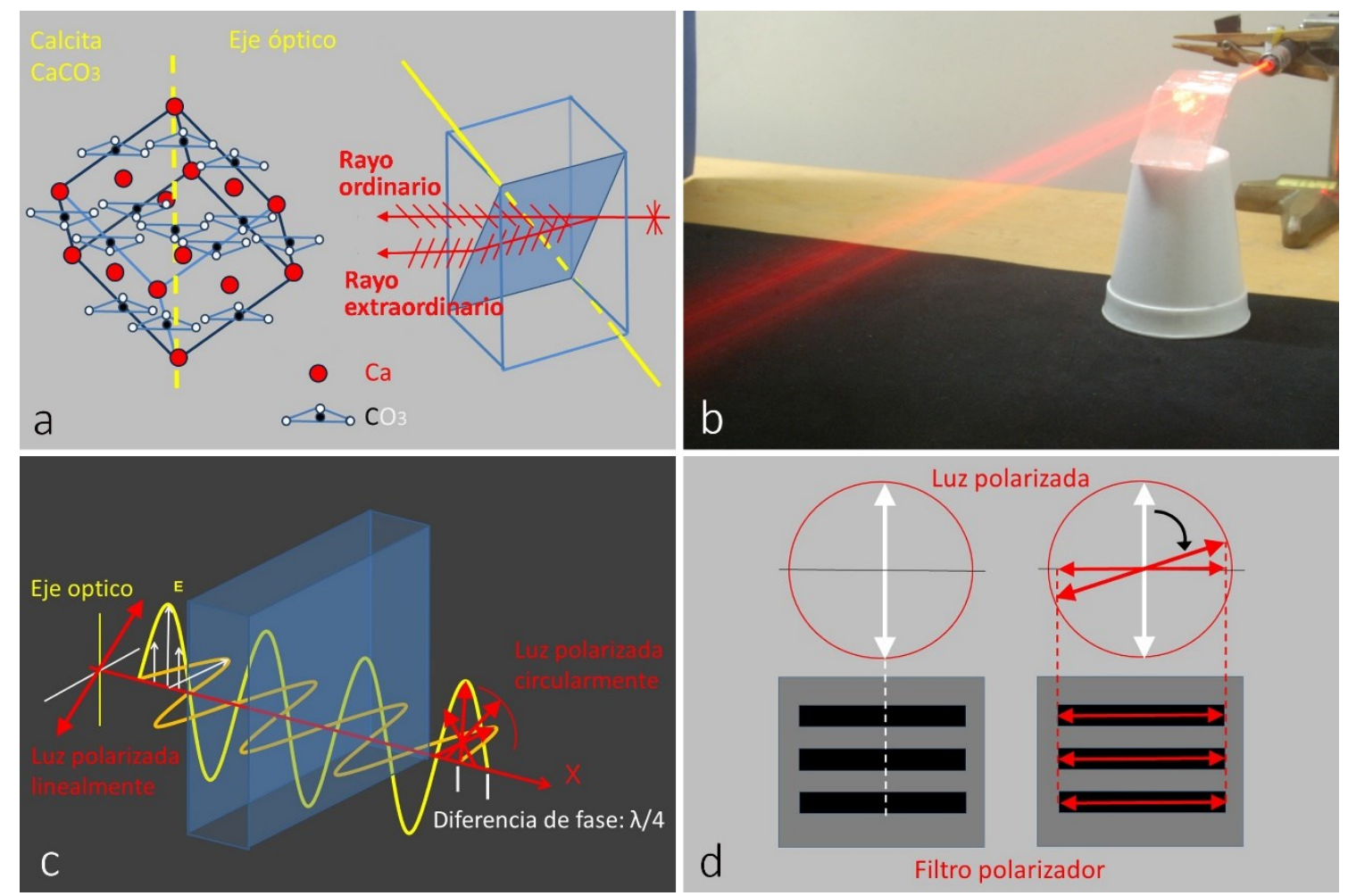

Fig. 12 - a) Estructura cristalina de la calcita con su eje óptico (en amarillo). b) Un rayo no polarizado, incidente en un cristal de calcita, se divide en dos haces, el rayo ordinario, que sigue la ley de refracción de Snell, y un rayo extraordinario, que no sigue la ley habitual de refracción. c) Si el grosor del material no es lo suficientemente grande, es posible que los dos rayos no se dividan, pero los dos componentes crean una onda resultante con la polarización cambiada en comparación con la inicial. En este ejemplo, la polarización lineal se transforma en polarización circular. d) Un polarizador bloquea la luz polarizada en la dirección ortogonal a su eje de transmisión. Al colocar un material birrefringente frente al polaroid, el material puede cambiar la polarización inicial, creando un componente de ésta en la dirección del eje de transmisión. De esta manera, la luz puede pasar a través del filtro.

Por ejemplo, si el retardo de fase es $\Delta \phi=\pi / 2$, se puede obtener una onda saliente polarizada circularmente. Este retraso de fase corresponde a una onda componente retardada con respecto a la otra, espacialmente, de $\Delta x=\lambda / 4$ (de hecho: $\Delta \phi=k \Delta x=(2 \pi / \lambda)(\lambda / 4)=$ $\pi / 2$ ). En general, el espesor del material, la longitud de onda de la luz y el índice de refracción, inciden en el retardo de fase, así como en el cambio de la polarización. Esta última propiedad es usada en óptica para obtener cualquier tipo de luz polarizada, a partir de una polarización lineal. Las Fig. 13 muestran el simple equipo experimental utilizado en los experimentos: una pantalla de computadora portátil es la fuente de la luz polarizada (FAKHRUDDIN, 2008). 

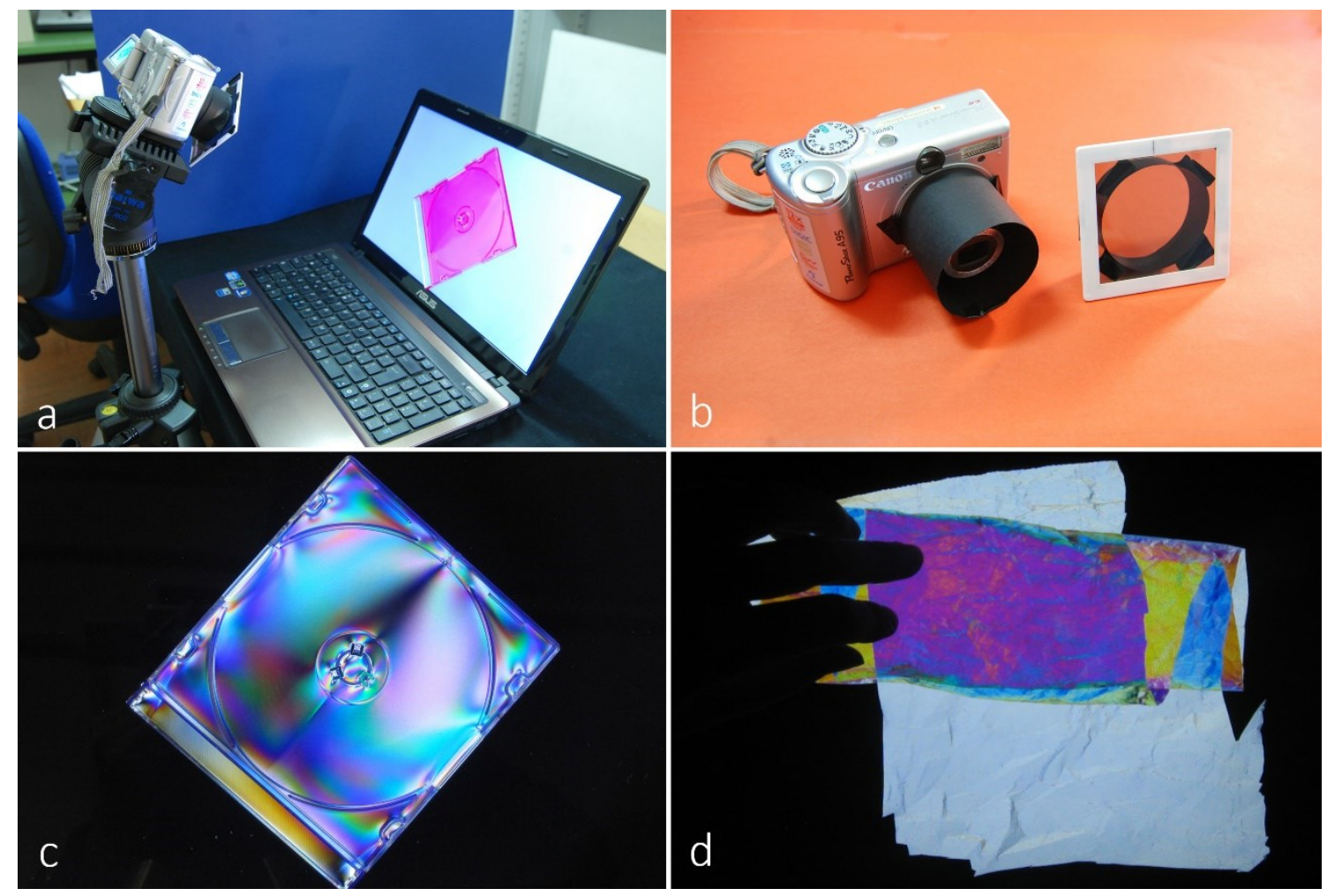

Fig. 13 - El equipo utilizado: a) una pantalla de computadora portátil que emite luz polarizada linealmente, b) una cámara y un filtro polarizador pegado delante de la lente de la cámara. c) Una cubierta de CD, en frente a la pantalla, muestra birrefringencia. En la imagen d), se puede observar cómo los colores de la luz polarizada, que pasan a través del segundo filtro, dependen del espesor del material, en este caso el celofán.

Así como el celofán, son birrefringentes las películas de polipropileno orientado y el plástico bajo tensión mecánica. Por lo general, el plástico es un material isotrópico, pero cuando es sometido a estrés, sus moléculas se orientan y se vuelve birrefringente, perdiendo la isotropía. Esta propiedad es aplicada en ingeniería mecánica para detectar el estrés de estructuras o componentes mecánicos. Se crean modelos plásticos de estos elementos, se colocan las maquetas bajo carga y se observan entre dos filtros polarizadores cruzados. Cuando los modelos son iluminados con luz blanca polarizada, la luz que pasa a través del segundo filtro, también dependerá del estrés, que causa variaciones de la polarización.

De esta forma, la imagen da información sobre la distribución de la tensión en las maquetas. Si el filtro de polarización está orientado para detener la luz de la computadora portátil, la pantalla se ve oscura. Sin embargo, si se colocan, entre la pantalla y el filtro, materiales capaces de cambiar la orientación de la polarización de la pantalla, es posible ver de nuevo la luz (Fig. 12d). El material cambia la polarización en función de su espesor y la longitud de onda de luz, haciendo visibles una gran cantidad de hermosos colores. Los estudiantes pueden crear imágenes espectaculares, utilizando material de reciclaje. 

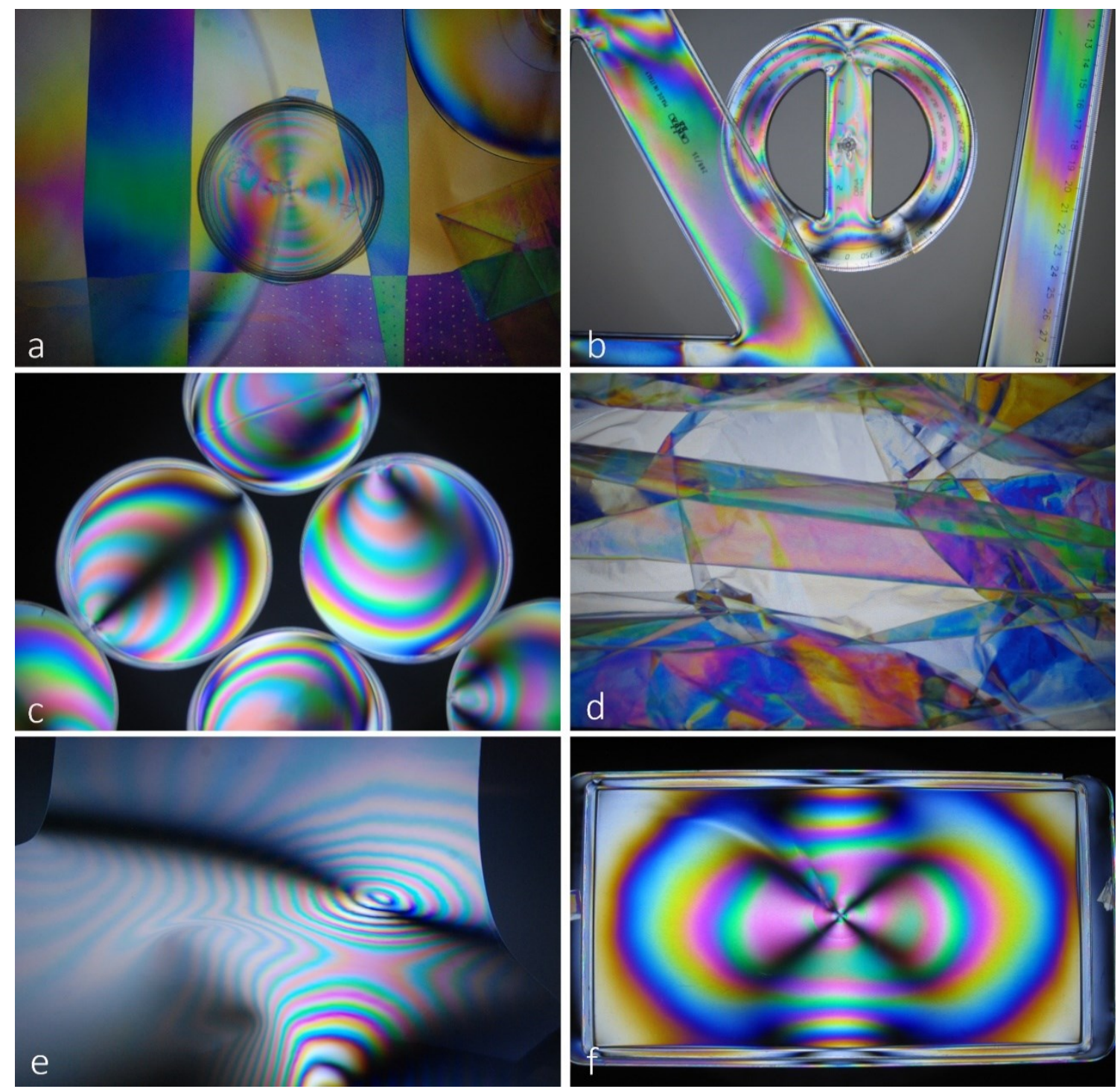

Fig. 14 - En esta figura se muestran varios ejemplos de birrefringencia (o de arte con basura): a) birrefringencia de películas de celofán sobrepuestas y una tapa de plástico, b) birrefringencia en "la escuela" c) birrefringencia de varios contenedores de plástico, d) de películas de polipropileno, e) de una lámina de plástico, f) de un contenedor de plástico.

En las Fig. 13c, d y 14, se pueden observar imágenes muy llamativas, que se obtienen colocando entre polaroid cruzados varios materiales tales como el celofán, polipropileno, plexiglás y plástico.

\section{Experimentos dulces}

En 1848, Louis Pasteur, biólogo y químico francés, descubrió que una solución de ácido tartárico rota la polarización de la luz. Pasteur también demostró la existencia de la quiralidad molecular y la isomería (PYE, 2001). La isomería es la propiedad de aquellos 
compuestos químicos que tienen igual fórmula molecular, pero son diferentes en sus estructuras y propiedades (es decir, las sustancias tienen los mismos átomos, pero en diversa disposición). En la quiralidad molecular, dos moléculas tienen la misma fórmula química, pero son una la imagen especular de la otra, como las dos manos, derecha e izquierda (de hecho, la palabra quiralidad viene desde el griego chir, que significa mano).

Pasteur descubrió que la quiralidad puede tenerse en dos configuraciones: levorrotatoria y dextrorrotatoria. El científico francés encontró esta propiedad, mirando los dos tipos de cristales de ácido tartárico, que son uno la imagen especular del otro. Pasteur dividió los dos tipos de cristales y demostró que, en una solución, su comportamiento era diferente: los cristales eran ópticamente activos, pero de diferente manera. Por tal motivo, él imaginó que también a nivel molecular, las moléculas podrían existir con los mismos átomos, pero con simetría especular.

Una sustancia es ópticamente activa cuando rota la dirección de la polarización de la luz (Fig. 15a), y se denomina como dextrorrotatoria o levorrotatoria, si gira el plano de polarización en el sentido de las agujas del reloj o en sentido anti horario, con respecto a un espectador que observa la luz acercándose hacia él.

Se puede dar una explicación intuitiva de la existencia de actividad óptica de esta manera. Se ha observado que una onda polarizada linealmente puede pensarse como la suma de dos ondas, u oscilaciones ortogonales, en cada punto del espacio. Sin embargo, resulta que es posible construir esta onda, también como la resultante de dos ondas polarizadas circularmente, una en sentido horario y la otra en sentido anti horario (BETTINI, 2017).

En la naturaleza existen moléculas con forma helicoidal, como el ADN. En particular, el ADN tiene la estructura de una hélice dextrorsa. Cuando una onda electromagnética polarizada linealmente incide en esta molécula, el componente polarizado circularmente en sentido horario (dextrorso) actúa de manera diferente en el ADN que el componente polarizado circularmente en sentido anti horario. Al final, debido a las oscilaciones de las cargas inducidas por el campo eléctrico incidente, la suma resultante de la radiación inicial y la onda electromagnética creada por la molécula, es una onda que ha sufrido una rotación dextrorsa del plano de polarización (BARRON, 2004).

Otras moléculas, generalmente orgánicas, y no sólo con una estructura exactamente helicoidal, tienen la propiedad de actividad óptica. Además, el efecto es independiente de la orientación de las moléculas.

En los experimentos presentados sobre la actividad óptica, se usan sustancias baratas como la sacarosa (azúcar de caña) y fructosa (azúcar de la fruta). La sacarosa es dextrorrotatoria, y la fructosa levorrotatoria. Otra sustancia que se puede utilizar es la glucosa, que es dextrorrotatoria (por esta razón, esta también se llama dextrosa y la fructosa levulosa). Las moléculas de fructosa y glucosa son imágenes especulares una de la otra (Fig. 15b). 

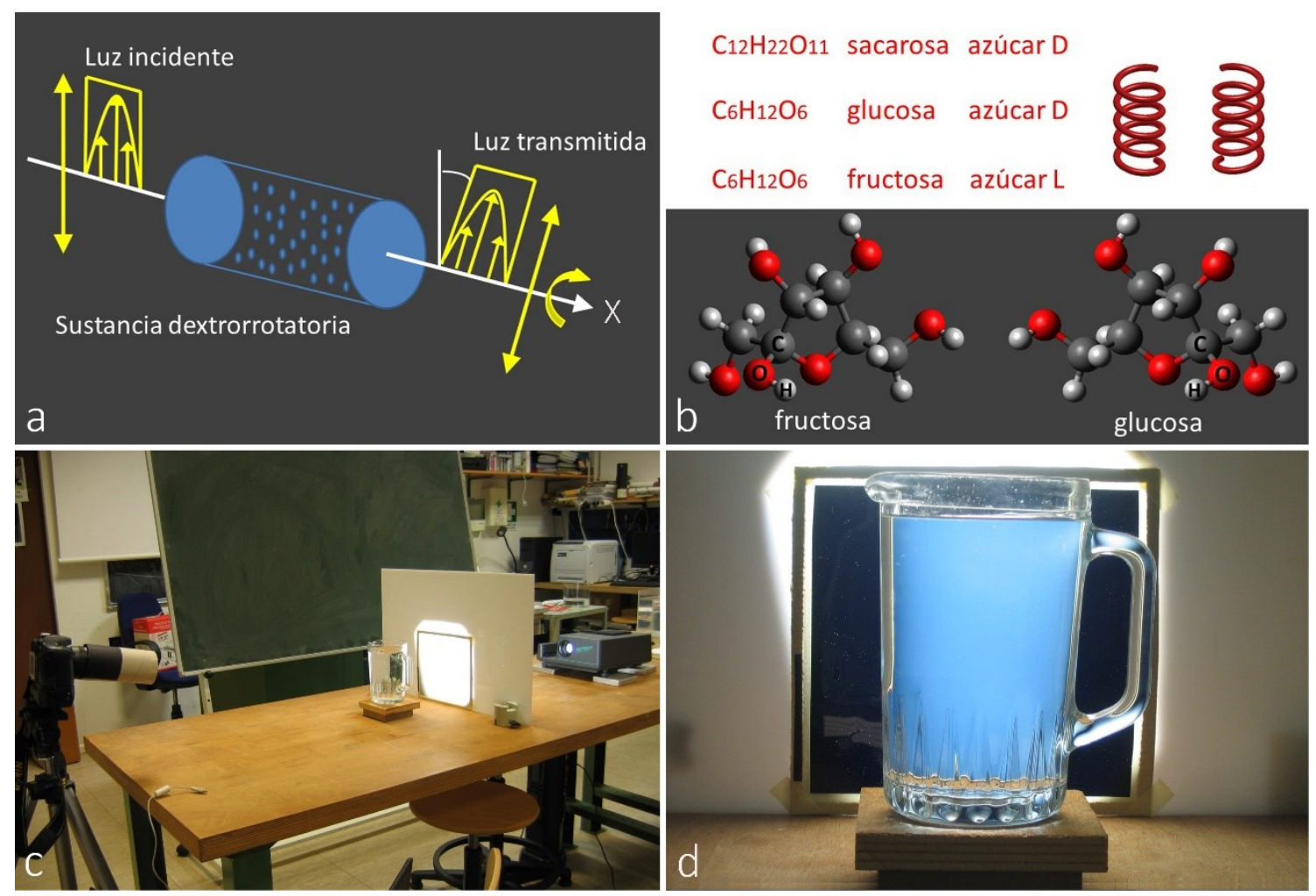

Fig. 15 - a) Imagen esquemática de la rotación de la polarización en una solución dextrorrotatoria. b) La glucosa y la fructosa son dos moléculas quirales. La glucosa es dextrorrotatoria (azúcar D), la fructosa es levorrotatoria (azúcar L), la sacarosa (azúcar de caña) es un azúcar D (dextrorrotatoria). c) El típico equipo usado en los experimentos: la luz polarizada proviene de un viejo proyector por diapositivas que tiene un filtro polarizador delante, y otro filtro es colocado en frente a la cámara. b) La imagen de una jarra llena de una solución de azúcar de caña entre los dos polarizadores cruzados.

Tal y como se observa en la Fig. 15c, se utilizó como lámpara un viejo proyector de diapositivas, un filtro polaroid para crear luz polarizada (también se puede usar una pantalla de laptop), otro filtro de polarización en frente a la cámara, para observar el efecto de la actividad óptica y frascos de vidrio de diferente forma para contener la solución ópticamente activa.

La rotación de la polarización depende de la concentración $c_{o}$ de la sustancia ópticamente activa en la solución, de la longitud del camino de la luz $\Delta x$ en la solución y de un factor de proporcionalidad $\gamma$ característico de la sustancia ópticamente activa, función decreciente de la longitud de onda $\lambda$. Si indicamos con $\alpha$ el ángulo de rotación de la polarización:

$$
\alpha=\gamma(\lambda) c_{o} \Delta x
$$

Por ejemplo, en la Fig. 15d, se puede observar lo que sucede cuando una jarra llena de solución de azúcar de caña, es ubicada entre dos polarizadores cruzados: la rotación de la 
polarización, debido a la actividad óptica, crea un componente del campo eléctrico a lo largo de la dirección de transmisión del segundo polaroid, y la luz puede pasar a través de este filtro. Como el ángulo de rotación de la polarización depende de la longitud de onda de la luz y por lo tanto del color, el filtro selecciona los componentes de la polarización que corresponden a ciertos colores, en el caso del experimento en la Fig. 15d, el color azul.

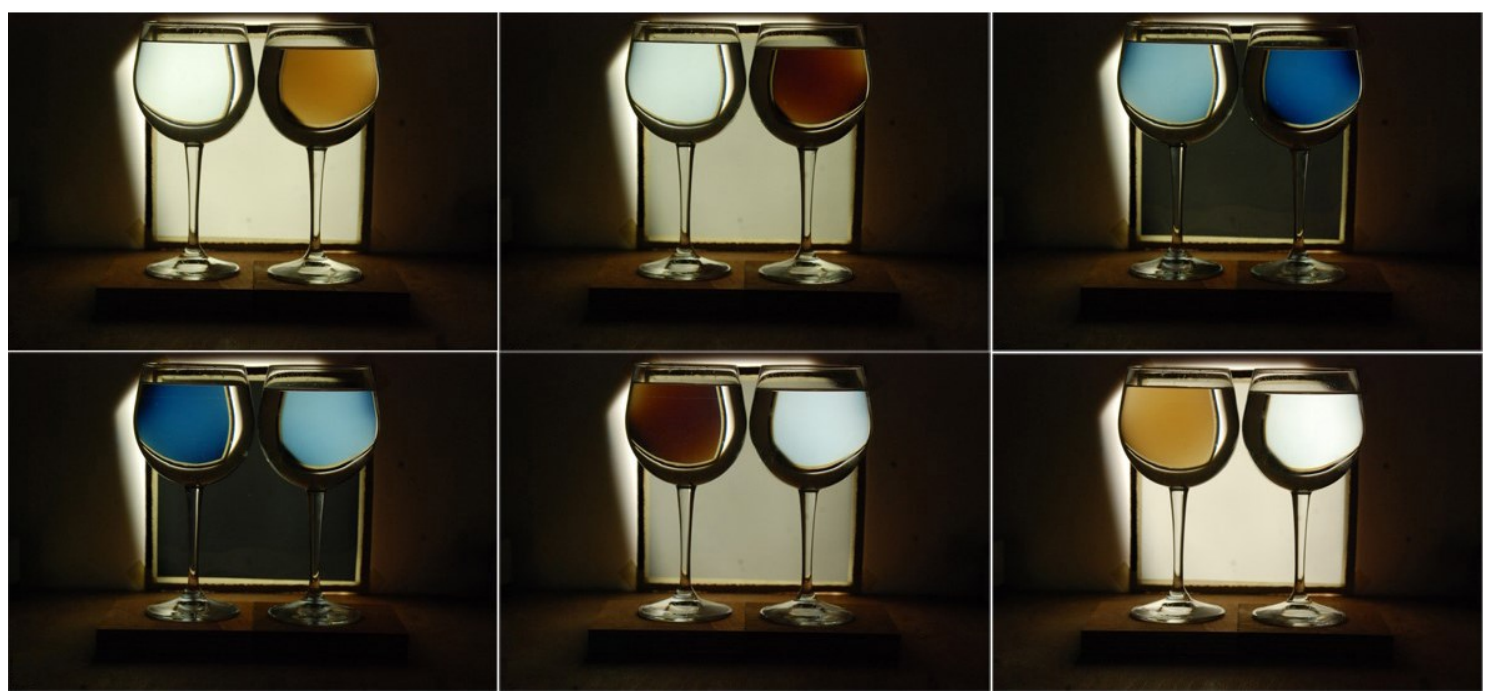

Fig. 16 - Rotación de la polarización de una solución de fructosa (a la izquierda) y de sacarosa (a la derecha). Cuando se rota el polaroid frente a la cámara, en sentido horario, es posible observar secuencias de colores en las dos soluciones, que son una opuesta a la otra. Esto se debe a que la sacarosa rota la polarización hacia la derecha (dextrorrotatoria) y la fructosa hacia la izquierda (levorrotatoria).

La fructosa y la sacarosa son sustancias con capacidad de actividad óptica muy parecida. Por ende, es interesante comprobar lo que ocurre, si se colocan dos vasos llenos de una solución de fructosa y de sacarosa de igual concentración entre los dos filtros (Fig. 16). Se puede rotar lentamente el segundo filtro, en sentido horario con respecto al observador, comenzando desde una situación en la que los dos polarizadores tienen ejes de transmisión paralelos. Se observa que la solución de sacarosa (a la derecha) aparece primero de color amarillo-rojo y después azul. Este efecto se puede explicar, teniendo en cuenta que la polarización que corresponde a una longitud de onda más grande, como la del color rojo, es rotada menos que la polarización asociada con longitudes de onda más pequeña, como la del color azul, por la dependencia de la función $\gamma=\gamma(\lambda)$ de $\lambda$. Para la solución de fructosa, también aparece una secuencia de colores, todavía opuesta a la de sacarosa, siendo la sacarosa dextrorrotatoria y la fructosa levorrotatoria.

La actividad óptica se utiliza en los sacarímetros, dispositivos que miden la concentración de azúcar en un líquido. Los sacarímetros se utilizan principalmente en la producción de vino, cerveza, jugos de frutas y azúcar de caña. 


\section{Metodología didáctica}

El laboratorio está diseñado principalmente para estudiantes de escuela secundaria o de los primeros años universitarios (aunque incluso en la escuela básica es posible realizar algunos de los experimentos propuestos con los niños, obviamente con diferentes objetivos de aprendizaje). Al ser un laboratorio de física, el objetivo es que los estudiantes aprendan los conceptos fundamentales relacionados con la polarización de la luz en física y sus aplicaciones en otras disciplinas.

Como prerrequisitos del laboratorio, se requiere que el alumno conozca los principales conceptos relacionados con las propiedades de las ondas, como frecuencia y longitud de onda, las propiedades básicas de los campos eléctricos y magnéticos, algunos conceptos de óptica fundamental, como las leyes de reflexión y refracción de la luz. La parte conceptual y matemática se puede desarrollar en mayor o menor medida, dependiendo del nivel de conocimientos básicos de los estudiantes, y si son estudiantes de secundaria o universitarios.

En las experiencias, los equipos y materiales utilizados son de bajo costo $\mathrm{y}$ fácilmente disponibles: se usan pantallas de computadoras como fuente de luz polarizada, filtros polarizadores, agua de caña de azúcar, fructosa y otros tipos de azúcares, y varios objetos reciclados como envases o cajas de plástico, los cuales son usualmente birrefringentes. En algunos experimentos se usa un simple puntero láser. Los filtros polarizadores, si no es posible comprarlos, se pueden recuperar desmontando las pantallas de antiguas laptop.

El laboratorio requiere aproximadamente 10 horas para su desarrollo conceptual y práctico. Esto puede ser una limitación, porque habitualmente, en los planes de estudio actuales, los profesores no disponen de mucho tiempo para dedicar a un tema. Los docentes podrían compensar la falta de tiempo, seleccionando solo algunos de los experimentos, o asignando algunas partes conceptuales o prácticas como tarea. Sería interesante que los profesores de física involucren a docentes de química y biología en el proyecto (esto podría ayudar con el tiempo disponible). Compartir el tiempo de laboratorio con profesores de otras disciplinas científicas, debería dejar en claro que la interdisciplinariedad no significa simplemente una descripción de las aplicaciones de la física a otras disciplinas, y que las divisiones del conocimiento son mucho menos definidas de lo que podría parecer al leer los currículos escolares: pensemos en la estrecha relación y los confusos límites entre la física y las matemáticas, entre la química y la física, o entre la química y la biología. La complejidad de la realidad requiere a menudo ser descrita con modelos en los que participen los aportes de diferentes disciplinas científicas.

En nuestro proyecto, la parte conceptual y experimental no se conciben como momentos de enseñanza y aprendizaje completamente distintos, como suele suceder en la práctica docente, más bien se enfatiza en una metodología donde no hay división entre teoría 
y práctica, pues estas se amalgaman y desarrollan en conjunto bajo la guía del docente, de acuerdo con un enfoque constructivista de la enseñanza (PAPERT, 1989).

La secuencia de los párrafos de este artículo, con su contenido, sigue la secuencia didáctica del laboratorio propuesto. Los estudiantes se dividen en grupos de trabajo de cuatro alumnos como máximo, a cada grupo se le asigna la tarea de encontrar los materiales necesarios para la experimentación y los experimentos se realizan con la orientación del profesor. La lección frontal tradicional debe ser limitada para maximizar la interacción entre los estudiantes y entre los estudiantes y el maestro. El docente puede orientar la práctica experimental y conceptual a través de cuestionarios o una serie de preguntas planteadas a los distintos grupos, para que los propios alumnos aprendan, de la forma más independiente posible, a través del razonamiento y la práctica de los conceptos científicos en cuestión. Para las partes más conceptuales, se puede pensar en utilizar la metodología de enseñanza del aula invertida. Al final de cada sección experimental, es posible solicitar a uno de los grupos que resuma lo que han aprendido, de forma oral o por escrito.

Una vez que los alumnos hayan adquirido los conocimientos básicos sobre la polarización de la luz, los profesores podrán afrontar la descripción de algunas aplicaciones tecnológicas muy comunes de la polarización, como las pantallas LCD, utilizadas en laptops, calculadoras, relojes digitales, y muchos otros dispositivos electrónicos. Por ejemplo, el docente puede sugerir ver o buscar videos en YouTube, relacionados con algunas aplicaciones tecnológicas de los polarizadores, como en las pantallas de las computadoras o los visores 3D. Algunos alumnos pueden ser los encargados de presentar y acompañar el visionado de los videos a toda la clase.

Algunas secciones del laboratorio se experimentaron parcialmente en dos jornadas de puertas abiertas de la Universidad Regional Amazónica Ikiam (Tena, Ecuador) y en algunos cursos de ingeniería de Física II de la misma universidad. En las jornadas de puertas abiertas de la universidad, algunos estudiantes de Física II y de las escuelas secundarias de Tena fueron previamente preparados por los docentes para presentar los experimentos ellos mismos a sus compañeros y a los visitantes. La retroalimentación cualitativa, en interés, participación y comprensión de los conceptos involucrados, tanto en la jornada de puertas abiertas como en los cursos de Física II, fue muy buena. Nos reservamos el derecho a realizar una experimentación cuantitativa más completa en el futuro.

\section{Consideraciones finales}

La polarización de la luz tiene diferentes aplicaciones y el estudio de este tema se presta muy bien a un enfoque de enseñanza interdisciplinar, desde la biología hasta la ciencia de los materiales. Este recurso pedagógico del docente, permite el desarrollo de una clase más atractiva, puesto que es posible mostrar ejemplos y aplicaciones de la vida real, captando así la atención de estudiantes que inicialmente no están interesados en física.

Así, por ejemplo, las abejas y otros animales explotan la luz polarizada para 
orientarse; entonces, los docentes pueden aprovechar esta circunstancia para hablar sobre la fisiología del ojo y el comportamiento animal, junto con la polarización de la luz por dispersión. Al mismo tiempo, es posible realizar en el aula experimentos de bajo costo y muy sugestivos. Cómo se ha visto, aparecen imágenes fantasmagóricas cuando se disponen algunos materiales entre dos filtros polarizadores cruzados, de ahí que los estudiantes puedan crear sus propias operas de arte. Además, esta podría ser una excelente ocasión para discutir en clase la relación entre arte, ciencia y tecnología y como las tendencias artísticas en el curso de la historia del arte moderna fueron muy dependientes de la ciencia y la tecnología. Por enunciar solo un ejemplo, la fotografía y el cine no desaparecieron a la pintura, más bien han dado una nueva herramienta a la comunicación y a la expresión de los sentimientos humanos, tal vez más emocionante que la pintura tradicional. Hoy en día, muchos artistas para crear sus obras de arte hacen un uso extensivo de la tecnología electrónica y digital, lo que requiere un amplio conocimiento de las tecnologías involucradas.

\section{Referencias bibliográficas}

ALONSO, M.; FINN, E. J. Física Volumen 2 - Campos y Ondas. Bogotá: Fondo Educativo Interamericano, 1970.

ATKINS, P.; DE PAULA J. Química Física. Buenos Aires: Médica Panamericana, 2008.

BARRON, L.D. Molecular Light Scattering and Optical Activity. Cambridge: Cambridge University, 2004.

BETTINI, A. A Course in Classical Physics 4 - Waves and Light. Berlin: Springer-Verlag, 2017.

DAVIDOVITS, P. Physics in Biology and Medicine. Amsterdam: Elsevier, 2008.

FAKHRUDDIN, H. Some Activities with Polarized Light from a Laptop LCD Screen. Physics Teacher, v. 48, n. 4, p. 229-231, 2008.

GHIRARDI G. Sneaking a Look at God's Cards: Unraveling the Mysteries of Quantum Mechanics. Princeton: Princeton University Press, 2007.

GUNTHER, L. The Physics of Music and Color. New York: Springer-Verlag, 2012.

HECHT, E. Óptica. Lisboa: Fundação Calouste Gulbenkian, 1991. 
JOHNSEN, S. The Optics of Life: A Biologist's Guide to Light in Nature. Princeton; Oxford: Princeton University Press, 2011.

KLAASSEN, R.G. Interdisciplinary education: a case study. European Journal of Engineering Education, v. 43, n. 6, p. 842-859, 2018.

LVOVSKY, A.I. Quantum Physics-An Introduction Based on Photons. Berlin: SpringerVerlag, 2018.

MCFADDEN, J.; AL-KHALILI, J. Life on the Edge: The Coming of Age of Quantum Biology. New York: Crown Publishers, 2014.

MICHELINI, M.; STEFANEL, A. A path to build basic Quantum Mechanics ideas in the context of light polarization and learning outcomes of secondary students. Journal of Physics: Conference Series, v. 1929, 012052, 1-11, 2021.

ODEH, S.; MCKENNA, S.; ABU-MULAWEH, H. A unified first-year engineering design based learning course. International Journal of Mechanical Engineering Education, v. 45, n. 1, p. 47-58, 2017.

PAPERT S.; Constructionism: A New Opportunity for Elementary Science Education - A proposal to the National Science Foundation. Cambridge: MIT, 1989.

PARKER, B. Good Vibrations: The Physics of Music. Baltimore: The Johns Hopkins University Press, 2009.

PASSINO, K.M. Biomimicry for Optimization, Control, and Automation. London: Springer - Verlag, 2004.

PYE, D. Polarised Light in Science and Nature. Bristol: IOP Publishing, 2001.

SAKURAI, J. J.; NAPOLITANO, J. Modern Quantum Mechanics. Cambridge: Cambridge University Press, 2012.

SICHERL-KAFOLA, B.; DENAC, O. The importance of interdisciplinary planning of the learning process. Procedia Social and Behavioral Sciences, v. 2, p. 4695-4701, 2010.

SPLINTER, R. Handbook of Physics in Medicine and Biology. Boca Raton FL: CRC Press, 2010 . 
TYNDALL, J. Six Lectures on Light. London: Longmans, Green and Co., 1882.

VON FRISCH, K. The Dancing Bees: An Account of the Life and Senses of the Honey Bee. London: Methuen \& Co. Ltd. 1966.

YÁNEZ E. et al. Física en la playa con Google Earth. Revista de Enseñanza de la Física, v. 30, n. 2. p. 109-118, 2018. 